\title{
Testing the state-dependent model of time perception against experimental evidence
}

\author{
Pirathitha Ravichandran-Schmidt ${ }^{1,2,3,4}$, Joachim Hass ${ }^{1,3,4}$ * $^{*}$ \\ 1 Faculty of Applied Psychology, SRH University Heidelberg, Heidelberg, Germany \\ 2 Faculty of Biosciences, University of Heidelberg, Heidelberg, Germany \\ 3 Bernstein Center for Computational Neuroscience (BCCN) Heidelberg/Mannheim \\ 4 Department of Theoretical Neuroscience, Central Institute of Mental Health, \\ University of Heidelberg/Medical Faculty Mannheim, Mannheim, Germany \\ * joachim.hass@srh.de
}

\begin{abstract}
Coordinated movements, speech and other actions are impossible without precise timing. Realistic computational models of interval timing in the mammalian brain are expected to provide key insights into the underlying mechanisms of timing. Existing computational models of time perception have only been partially replicating experimental observations, such as the linear increase of time, the dopaminergic modulation of this increase, and the scalar property, i.e., the linear increase of the standard deviation of temporal estimates. In this work, we incorporate the state-dependent computational model, which encodes time in the dynamic evolution of network states without the need for a specific network structure into a biologically plausible prefrontal cortex (PFC) model based on in vivo and in vitro recordings of rodents. Specifically, we stimulated 1000 neurons in the beginning and in the end of a range of different time intervals, extracted states of neurons and trained the readout layer based on these states using least squares to predict the respective inter stimulus interval. We show that the naturally occurring heterogeneity in cellular and synaptic parameters in the PFC is sufficient to encode time over several hundreds of milliseconds. The readout faithfully represents the duration between two stimuli applied to the superficial layers of the network, thus fulfilling the requirement of a linear encoding of time. A simulated activation of the D2 dopamine receptor leads to an overestimation and an inactivation to an underestimation of time, in line with experimental results. Furthermore, we show that the scalar property holds true for intervals of several hundred milliseconds, and provide a mechanistic explanation for the origin of the scalar property as well as its deviations. We conclude that this model can represent durations up to $750 \mathrm{~ms}$ in a biophysically plausible setting, compatible with experimental findings in this regime.
\end{abstract}

\section{Introduction}

The ability to estimate the passage of time is of fundamental importance for perceptual and cognitive processes [82] such as speech, music, [9] and decision making [7]. Impairments in time perception have been reported for various psychiatric disorders, such as schizophrenia, Parkinson's disease, Huntington's disease and major depression $[8,9,45,62,71]$. In mammalian neural circuits, time perception spans ten orders of 
magnitudes including millisecond timing, interval timing and circadian rhythms spanning an entire day $[9,63]$. Among them, interval timing ranges from a few hundred milliseconds to several minutes and is involved in various processes as for instance foraging [43], decision making [7], sequential motor performance [5] and associative conditioning $[9,27,63]$.

Despite the importance of telling time, the exact neural mechanisms of time perception are not fully understood yet. Proposing and testing biologically plausible computational models are a promising way to gain understanding of these mechanisms. Currently discussed models of time perception [37, 38] include the state-dependent network model [11], models of ramping activity [20] and synfire chains [40] as well as the striatal beat model $[60,64]$. Here, we focus on the state-dependent network model [11], in which timing is not encoded by a dedicated tuning mechanism, but by the intrinsic neuronal and synaptic properties [46]. Specifically, the author claims that the representations of time up to $400 \mathrm{~ms}$ are generated by time-dependent mechanisms within the network, such as short-term plasticity and slow inhibitory postsynaptic potentials (IPSPs) caused by GABA $_{B}$ synapses [11]. More recent theoretical studies suggest that the hidden states of the network, induced by, e.g., short-term plasticity, allow to encode any stimulus together with its temporal context and time itself in the neuronal activity $[13,14,46]$.

Here, we aim to test whether this model may pose a plausible neural mechanism of time perception in the brain. To this end, we first implement the model into a strongly data-driven spiking network model [41] including substantial heterogeneities in the neuron and synapse parameters. Biological constraints, and heterogeneity in particular, has previously shown to pose a challenge to simple computational models, e.g., of working memory [42]. Second, we test whether the model can reproduce established experimental findings of time perception and can thus be validated by empirical data. This work is part of an ongoing research project that tests all of the above mentioned models in the same way as we test the state-dependent network model here.

Timing is characterized by two fundamental properties, namely the linear psychophysical law for the average duration estimates [23], and the scalar property for timing errors, also referred to as Weber's law [9, 18, 29, 33, 57]. The linear psychophysical law states that real time is proportional to the averaged representation of time [23]. Although deviations from this linear relation have also been reported [24], calling for a power law generalization called Steven's law [24, 78], these deviations are small and the linear psychophysical law is still most widely accepted for time perception $[1,3]$. However, the linear relation is not always a one-to-one relation. Already in the 19th century, Vierordt found that short intervals are overestimated, while longer intervals are underestimated [30, 83]. The indifference point varies depending on the intervals considered [55], e.g. employing intervals within the range of $200-1000 \mathrm{~ms}$ show the indifference point to be between $600-800 \mathrm{~ms}$ with the slope of timing of $\sim 0.52$ [49], whereas testing intervals within the range of $400-2000 \mathrm{~ms}$, shifts the indifference point to higher values $(\sim 1400 \mathrm{~ms})$ with a timing slope of $\sim 0.86[26]$. Although some studies assume that the indifference point can be calculated by the arithmetic mean of the applied intervals [4, 26, 55], other studies do not support this hypotheses [55, 87, 89].

The scalar property refers to the observation that timing errors, or more specifically, the standard deviation of duration estimated between different trials, increase linearly with the duration of the interval to be timed. To quantify this relation, the Weber fraction can be used, dividing the standard deviation of the duration estimate by its mean. A constant Weber fraction over a range of durations is an indicator of Weber's law and has been observed in numerous studies. However, the scalar property is not universal, but seems to hold for intervals of around 200/400 ms [2, 25, 28, 32] up to $1200 / 2000 \mathrm{~ms}[28,86]$ in humans, with typical Weber fractions of around $0.05-0.13$ 
$[28,34]$. For shorter intervals, the Weber fraction decreases, indicating a sublinear (e.g. square-root) scaling of timing errors, while for longer intervals, a superlinear increase of the Weber fraction is reported (in humans, see $[28,86]$ and in animals $[2,15])$. During information-theoretical methods, Hass and Herrmann [39] suggested that these different scaling regimes of timing errors may indicate different mechanism underlying time perception. In particular, the scalar property is explained by so-called variance-based timing in this framework, i.e., using the temporal information contained in the timing errors itself, which increase over time and thus, can be used to decode time by means of a pure diffusion process.

While the linear psychophysical law and the scalar property constitute static properties of time perception, our sense of time is also highly variable in different situations and strongly depends on a number of factors, including emotions and mood [18], attention [69] and stimulus properties [21, 50, 85]. A prominent pharmacological modulator of time perception is dopamine $[9,72,79]$. Specifically, studies suggest that from the two main types of dopamine receptors, namely D1 and D2 receptor, the latter one has a high potential to modify the pacemaker speed [61]. Several studies report that a decreased dopaminergic activity, e.g., by dopaminergic antagonists, can slow down the internal clock leading to an underestimation of timing intervals, and increasing levels of dopamine, e.g., by dopaminergic agonists, speeds up the internal clock [9, 72, 79]. Furthermore, studies involving patients suffering from psychiatric disorders involving dopaminergic disbalance, such as schizophrenia [45, 62] and depression [8, 19], demonstrate altered performance in time discrimination tasks compared to healthy subjects [71]. A meta-analysis published by Thoenes and Oberfeld in 2015 shows a significantly reduced speed of the perceived flow of time in depressive subjects compared to non-depressive subjects [79]. Another study reveals that the positive symptoms of schizophrenia are related to an overestimation of timing intervals, leading to a speeding up of the internal clock [80].

To model the mechanisms of timing, the exact anatomical location of timing needs to be known. For this, fMRI studies suggest the involvement of prefrontal cortex, basal ganglia and striatum in timing processes ([56]). Various other studies are pointing towards the prefrontal cortex (PFC) especially in humans $[47,53]$ and also in animals $[10,52,67,88]$. As the indications suggest an involvement of the PFC in timing, in this work, we implemented the state-dependent network model, proposed by Buonomano [11], within a strongly data-driven network model of the PFC [41] and tested whether this model can reproduce established experimental findings of timing, namely linear timing, scalar property and the dopaminergic modulation. Since the model successfully reproduces all three properties, we further analyzed the underlying mechanisms on the basis of the trained readout weights and the synaptic currents of the selected neurons for each interval. Thereby we determined that the onset of the spike time of the neurons is mostly driven by their synaptic currents, e.g. short term plasticity and inhibition. Additional ablation studies confirm this hypothesis. Furthermore, limitations for longer interval durations and higher Poisson noise of up to a firingrate of $1000 \mathrm{~Hz}$ are examined to test the robustness of the model.

\section{Results}

\section{Reproduction of experimental results}

In order to test timing properties in a more realistic model, we incorporated the state space model proposed by Buonomano [11] into the prefrontal cortex (PFC) [41] which

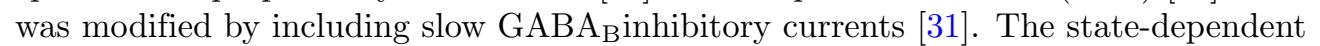
PFC model, cf. Fig. 1, is stimulated in the beginning and in the end of an interval with 
a step current, extracted the states of all neurons within a time window and trained the readout neurons following the readout mechanisms of a liquid state machine [48, 58]. We trained the weights of the readout units with various inter stimulus intervals (50$750 \mathrm{~ms})$ in $50 \mathrm{~ms}$ steps and tested them with intervals $(50-750 \mathrm{~ms})$ in $25 \mathrm{~ms}$ steps. The normalized outputs of the trained readout units for different test intervals are shown in Fig. 2A.

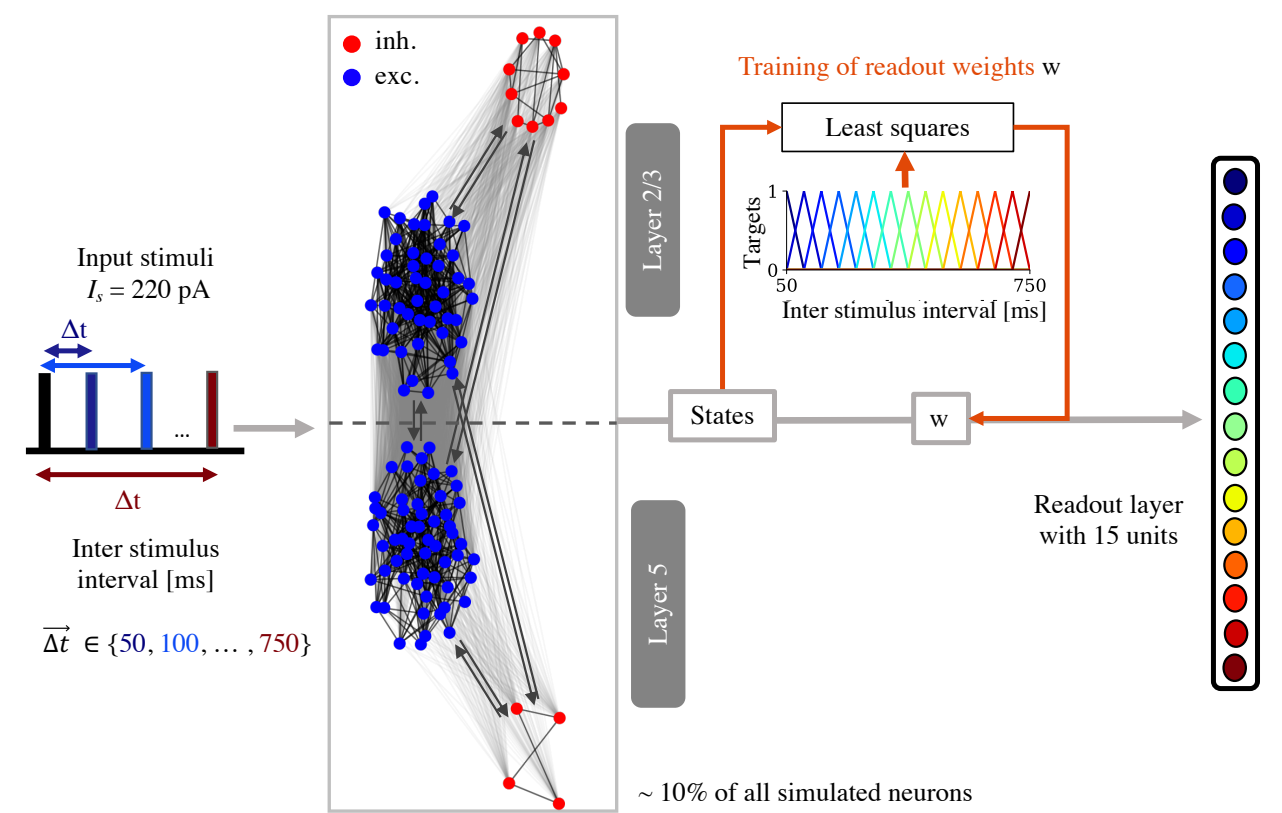

Fig 1. Illustration of the interval timing model. The gray box in the middle illustrates the PFC network model from [41] (only $10 \%$ of all neurons) with layer $2 / 3$ and layer 5 excitatory (blue) and inhibitory (red) neurons and their connectivities (gray arrows). This full network is stimulated (left to the gray box) in the beginning and the end of several interstimulus intervals (color-coded from $50 \mathrm{~ms}$ to $750 \mathrm{~ms}$ ). Afterwards, states were generated within a window from the spiketrains, and the readout weights to the readout layer (same colors and number as interstimulus intervals) were trained via least-squares with the targets and states as illustrated on the right. Training is depicted with orange arrows.

Each readout unit shows a tuning curve for its specifically trained intervals, also allowing the model to generalize to intervals it has not seen during training. Testing an inter-stimulus interval in between two trained intervals, e.g. $125 \mathrm{~ms}$, leads to a co-activation of the $100 \mathrm{~ms}$ and $150 \mathrm{~ms}$ readout unit. For longer test intervals, the tuning of the outputs becomes broader. The averaged subjective time, which is calculated by multiplying the output values of the readout units with their corresponding intervals and summing up over all readout units can be seen in Fig 2B. Equivalent to the linear psychophysical law from experimental studies, the estimated time is well described by a straight line, cf. Fig $2 \mathrm{~B}$, with a fit error of $\mathrm{RMSE}_{\mathrm{t}}=49.3 \pm 10.5 \mathrm{~ms}$. The subjective time for each interval over 50 trials was statistically verified by an one-way ANOVA $(F=1198, p<0.001)$. In agreement with Vierordt's law, we observe an overestimation of shorter and an underestimation of longer interval, cf. 2A. Fitting the estimated time, we get $y=0.83( \pm 0.05) \cdot x+69.9( \pm 16.02)$ of which the slope, which is in the range of the experimental observations [26, 49], significantly differs from 1.0 (one sample t-test: $t$ $\left.=-24.06, p<0.001, \mathrm{R}^{2}=0.97 \pm 0.02\right)$. The indifference point of the averaged estimated time can be found at $\mathrm{IP}_{\mathrm{avg}}=523 \mathrm{~ms}$ in line with experimental studies [87], 
which is larger than the arithmetic mean of the intervals with $400 \mathrm{~ms}$.

The evolution of the standard deviations of the estimated time over 50 trials, as shown in Fig $2 \mathrm{~B}$ and $\mathrm{C}$, are examined by fitting the data to a linear and a square-root function, for which the root-mean squared error is $\mathrm{RMSE}_{1}=3.5 \mathrm{~ms}$ and $\mathrm{RMSE}_{\mathrm{s}}=4.3 \mathrm{~ms}$ respectively. The trial averaged Weber fraction of 0.059 within the range of 400 $750 \mathrm{~ms}$, cf. green line in Fig. 2D, matches experimental observations [28, 36]

A
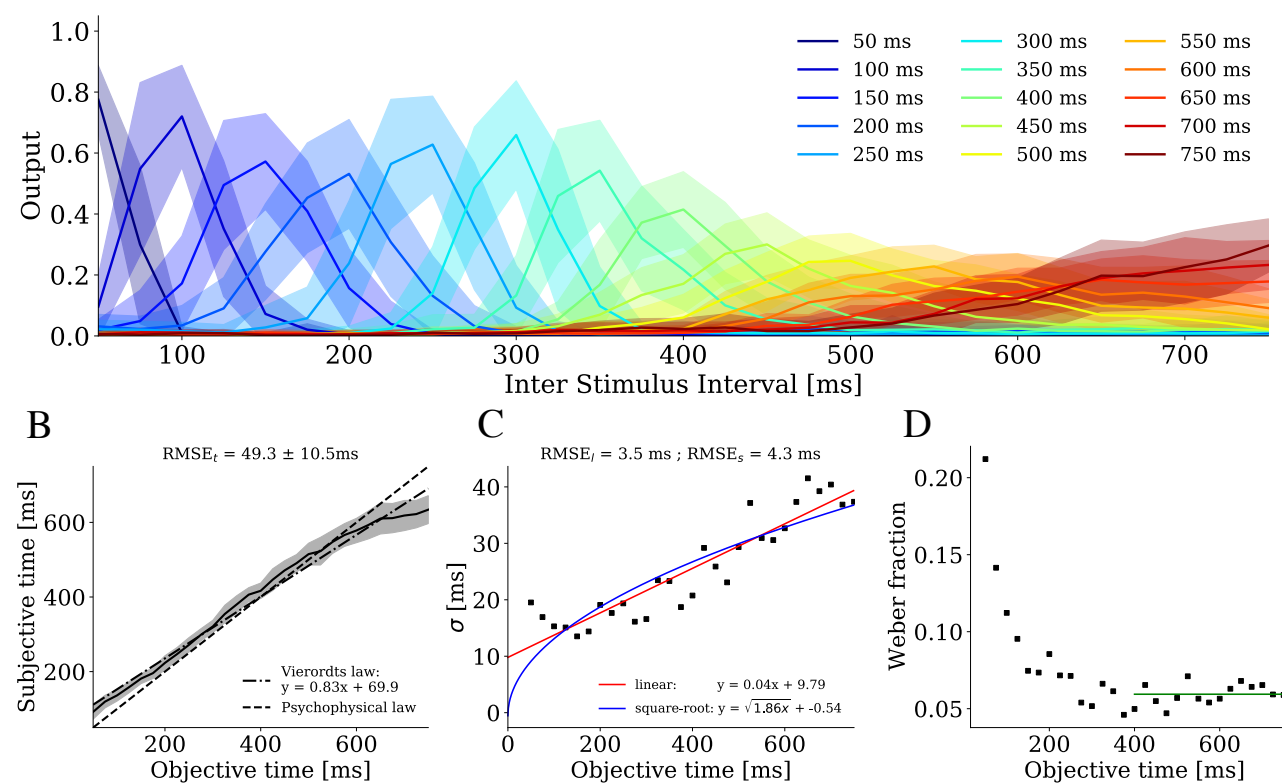
$\mathrm{C}$
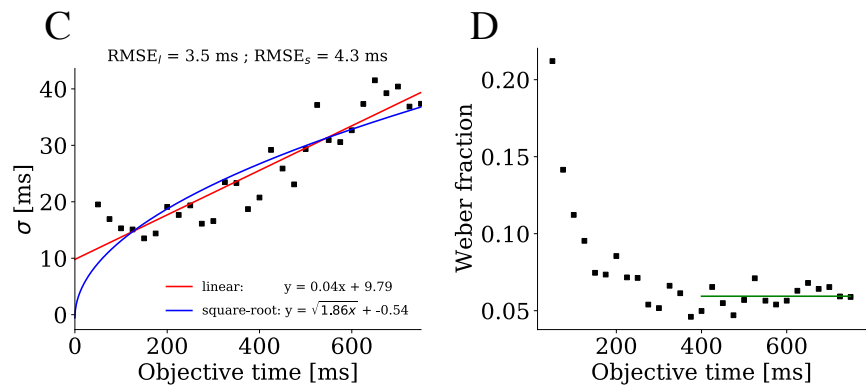

Fig 2. Psychophysical law and scalar property of the state-dependent PFC model

A Illustration of the averaged outputs and the standard deviations of each trained readout unit (color-coded) for test intervals (x-axis). B The averaged subjective time over 50 trials after multiplying the outputs with their corresponding trained interval is shown here with the standard deviations (shaded region shows corresponding standard deviations). The dashed black line indicates the expected objective time. Linear fit of estimated time to test the Vierordt law is plotted as dashed-dotted line. C Standard deviation of the estimated time are better described by a straight line (red) compared to a square-root function (blue), which is in line with the scalar property. RMSE $\mathrm{E}_{1}$ and $\mathrm{RMSE}_{\mathrm{s}}$ are calculated to both fits. D The trial averaged Weber fraction is fitted by a flat line in the range $400-750 \mathrm{~ms}$ with a constant value of 0.059 well in line with experimental observations [26, 49].

Both properties, namely the linear timing and the scalar property, were validated by retraining the model with newly initialized neuronal and synaptic parameters drawn from experimental validated distributions as in [41]. Across ten different parameter sets, the outcome of the psychophysical law yields a timing error of $\mathrm{RMSE}_{\mathrm{t}}=49.1 \pm 4.8 \mathrm{~ms}$, with a Vierordt slope of $0.85 \pm 0.04$ and the averaged indifference point at $545.5 \pm$ $23.3 \mathrm{~ms}$. Similarly, the averaged standard deviations describing Weber's law can be linearly fitted by $y=0.04( \pm 0.01) \cdot x+13.88( \pm 1.55)$ with an $\mathrm{RMSE}_{1}=4.44 \pm 0.95 \mathrm{~ms}$. Fitting the standard deviations by a square-root function resulted in $y=\sqrt{1.91( \pm 0.71) \cdot x}+2.56( \pm 2.93)$ with a slightly better $\mathrm{RMSE}_{\mathrm{s}}=4.20 \pm 0.81 \mathrm{~ms}$.

In order to examine the modulation of the D2 receptor, we changed the neuronal and synaptic parameters according to Table 6 for the test phase, while for training the 
A

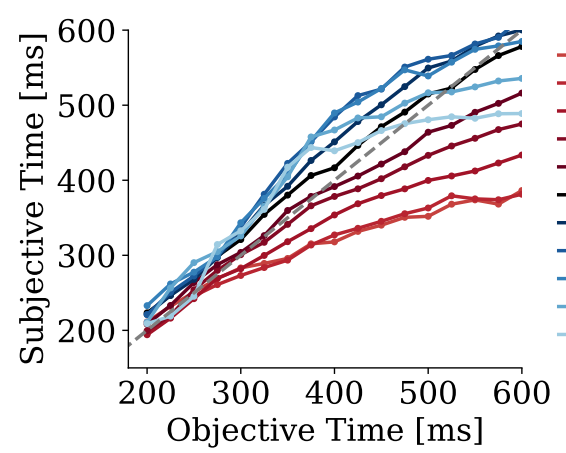

B

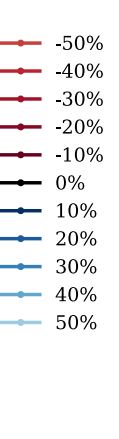

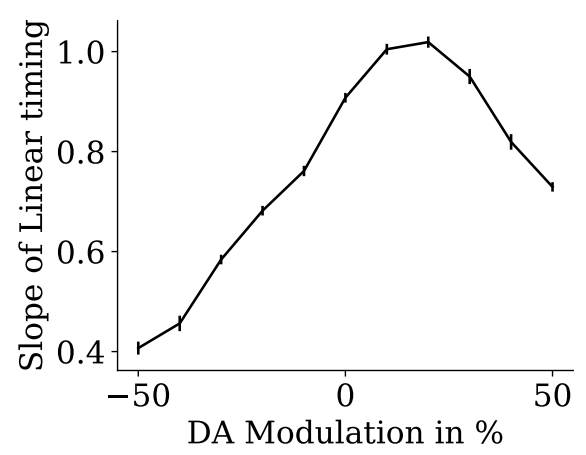

Fig 3. Effects of dopaminergic modulation

A Linear timing with respect to unmodulated estimation (black) for agonistic (blue) and antagonistic dopamine modulation of \pm 10 to $\pm 50 \%$ within the range of 200 $550 \mathrm{~ms}$. B The slope of the linear timing is calculated for each percentage of modulation (standard error depicted by error bars).

readout weights parameters were the same as before. The altered time estimates for DA modulation from $0 \%$ to $\pm 100 \%$ for the test intervals of $50-750 \mathrm{~ms}$ are presented in Suppl. Fig. 1 in Supplementary Materials. As both agonistic and antagonistic modulation show boundary effects for DA modulations $> \pm 50 \%$ and for longer and shorter interval (Suppl. Fig. 1 in Supplementary Materials), we only considered intervals within a range of $200 \mathrm{~ms}-600 \mathrm{~ms}$ and DA modulations up to $\pm 50 \%$, see Fig $3 \mathrm{~A}$. Within this range, subjective time shortens for higher antagonistic modulations (from darker to brighter red colors), and lengthens for agonistic modulation (from darker to brighter blue colors). For agonistic modulations $>20 \%$, the model fails to estimate time. Additionally, the slopes of the estimated time reach their maximum around $20 \%$ and drop again for higher values, cf. Fig. 3B. The slopes of the linear timing over 50 trials were statistically verified by an oneway ANOVA $(\mathrm{F}=280.4$, $p<0.001)$. The modulation did not show a change in the standard deviation consistent with the literature, see Suppl. Fig. 2 in Supplementary Materials.

If we retrain the readout weights on agonistic and antagonistic modulation of $30 \%$, we can counteract the changing speed of the internal clock. Fig.4 shows the psychophysical law and the Weber's law for agonistic dopaminergic modulation of $30 \%$ (red, $\mathrm{RMSE}_{\mathrm{t}}=51.0 \pm 8.6 \mathrm{~ms}, \mathrm{RMSE}_{\mathrm{l}}=2.9 \mathrm{~ms}$ and $\mathrm{RMSE}_{\mathrm{s}}=3.3 \mathrm{~ms}$ ), for the antagonistic dopaminergic modulation of $-30 \%$ (blue, $\mathrm{RMSE}_{\mathrm{t}}=54.1 \pm 11.3 \mathrm{~ms}$, $\mathrm{RMSE}_{\mathrm{l}}=3.7 \mathrm{~ms}$ and $\mathrm{RMSE}_{\mathrm{s}}=3.7 \mathrm{~ms}$ ) and for the original case from Fig. 2B (black). As a chronic activation of the dopaminergic system during, e.g. addiction, lead to an adaptation by neuroadaptive processes $[70,77]$, this is in line with our results in which a retraining of the readout weights can be compared to an adaptation of the network during chronic application.

\section{Limitations of the model}

In order to examine the limitations of the model, we trained for interval durations up to $2000 \mathrm{~ms}$ and tested within the same range. Doing so, we determined a degraded time perception which conforms with the theory of Vierordt (slope $=0.66 \pm 0.07$, one sample $\mathrm{t}$-test to compare to the identity $(\mathrm{t}(49)=-35.57, p<0.001), \mathrm{IP}_{\mathrm{avg}}=1099.2 \mathrm{~ms}$,

$R^{2}=0.83 \pm 0.07$, oneway ANOVA for each estimated time across 50 trials: $\mathrm{F}=361.6$, $p<0.001)$. Calculating the error to the expected time yields $\mathrm{RMSE}_{\mathrm{t}}=264.0 \pm 43.4 \mathrm{~ms}$, cf. Fig. 5A. The error of the standard deviation to the linear fit $\left(\mathrm{RMSE}_{1}=23.5 \mathrm{~ms}\right)$ is 
A

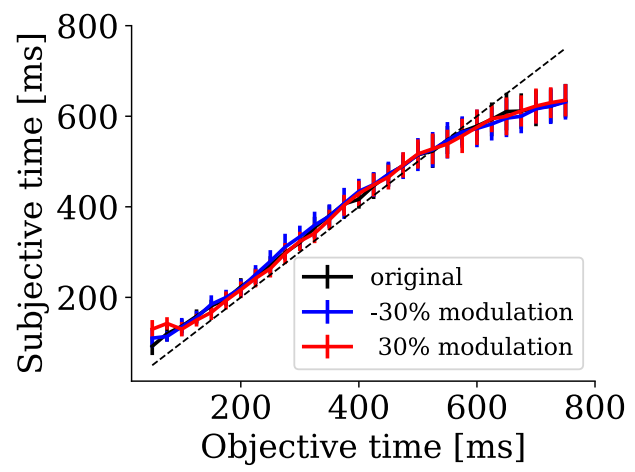

B

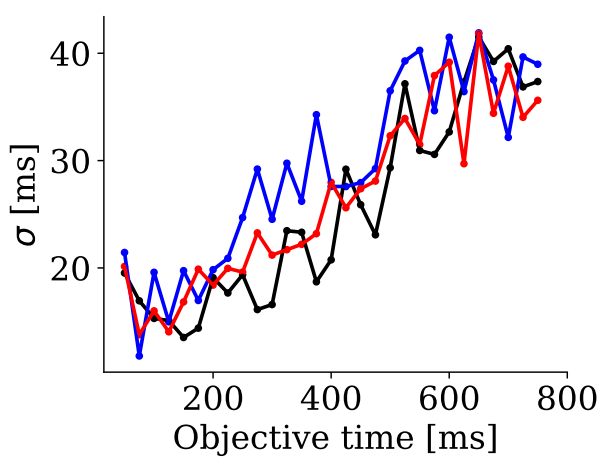

Fig 4. Retraining after mild dopaminergic modulation recovers linear timing and scalar property.

A,B Estimated times and the corresponding standard deviations over 50 trials are shown for the agonistic modulation of $30 \%$ (red) and for the antagonistic modulation of $-30 \%$ (blue). The estimated time and the standard deviation from Fig. 2 are shown in black for comparison.

indistinguishable from square-root fit $\left(\mathrm{RMSE}_{\mathrm{s}}=21.1 \mathrm{~ms}\right)$, cf. Fig. $5 \mathrm{~B}$.

Table 1. Parameters for different noise levels with $1000 \mathrm{~Hz}$ Poisson neurons

\begin{tabular}{ccccccc}
$\begin{array}{c}\mathrm{I}_{\text {back }} \text { reduction } \\
{[\%]}\end{array}$ & $\begin{array}{c}\text { weights } \\
{[\mathrm{nS}]}\end{array}$ & $\begin{array}{c}\mathrm{RMSE}_{\mathrm{t}} \\
{[\mathrm{ms}]}\end{array}$ & slopes & $\begin{array}{c}\mathrm{IP}_{\text {avg }} \\
{[\mathrm{ms}]}\end{array}$ & $\begin{array}{c}\mathrm{RMSE}_{\mathrm{l}} \\
{[\mathrm{ms}]}\end{array}$ & $\begin{array}{c}\mathrm{RMSE}_{\mathrm{s}} \\
{[\mathrm{ms}]}\end{array}$ \\
\hline \hline $\begin{array}{c}0 \% \\
\text { (orig. 1 Hz) }\end{array}$ & 0.5 & $49.3 \pm 10.5$ & $0.83 \pm 0.05$ & 520.0 & 3.5 & 4.3 \\
$-7 \%$ & 0.003 & $69.3 \pm 17.6$ & $0.74 \pm 0.08$ & 472.1 & 4.1 & 4.4 \\
$-21 \%$ & 0.005 & $90.5 \pm 23.4$ & $0.67 \pm 0.11$ & 512.5 & 7.1 & 5.9 \\
$-36 \%$ & 0.007 & $107.9 \pm 25.6$ & $0.56 \pm 0.12$ & 477.7 & 6.3 & 4.6 \\
$-50 \%$ & 0.010 & $120.1 \pm 30.2$ & $0.52 \pm 0.14$ & 458.7 & 10.2 & 8.2
\end{tabular}

As a typical cortical neuron receives connections from approximately 1000-10 000 other neurons $[6,65]$, and we, so far, only tested with 10 Poisson neurons each with $f=1 \mathrm{~Hz}$, We also examined whether time perception works at higher noise levels. We increased the firing rates of the 10 Poisson neurons up to $1000 \mathrm{~Hz}$ and compared timing for different noise levels by increasing the weights of the Poisson neurons and simultaneously reducing the background current to avoid overexcitation and to keep the standard deviation of the sub threshold membrane potential constant, cf. Table 1 and Fig. 5C and D.

In our experiments, we find worse time estimation for increased noise levels by higher weights on the $1000 \mathrm{~Hz}$ Poisson neurons. The linear timing error increases and Vierordts law becomes pronounced, see slopes, indifference points and $\mathrm{RMSE}_{\mathrm{t}}$ in Table 1. Webers law only holds true for low noise levels, see RMSE $E_{l}$ and $\mathrm{RMSE}_{\mathrm{s}}$ in Table 1. For higher noise levels the standard deviations are better described by the square-root function, cf. Fig. 5B and Table 1, but in general, the error for both, linear and square-root fit, becomes larger. 
A
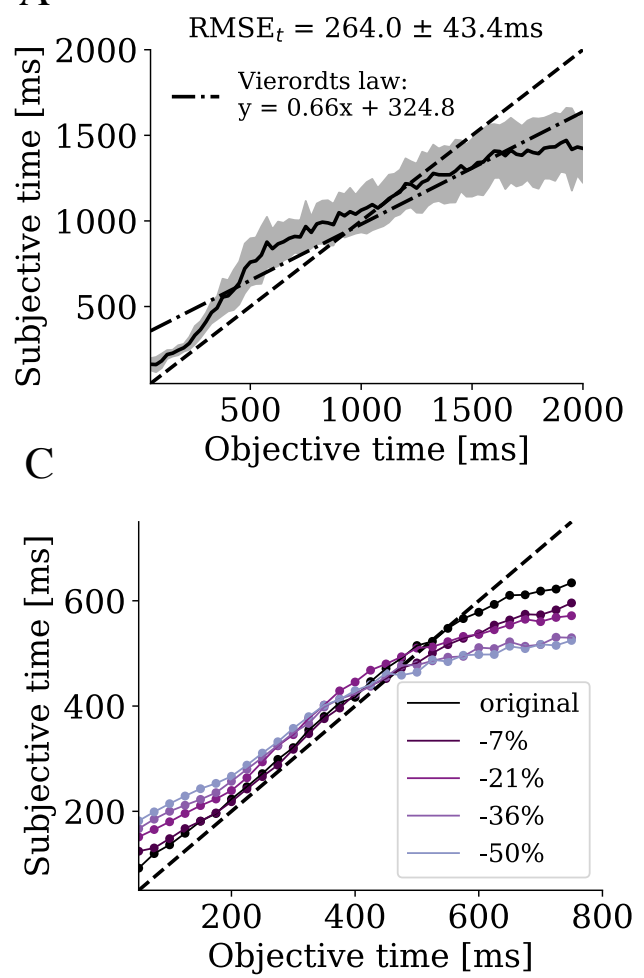

B
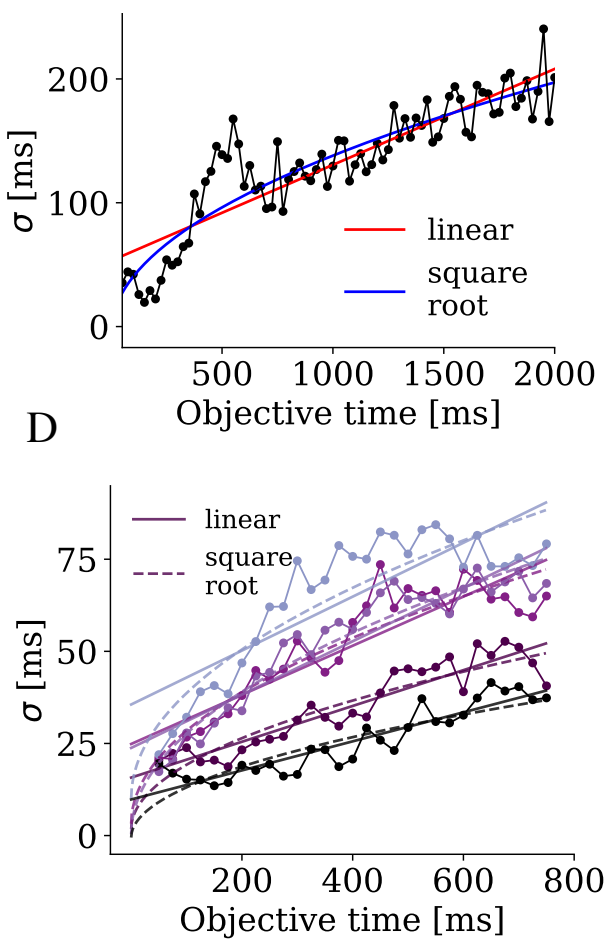

Fig 5. Test for limitations within the network model.

A, B The estimated time and the standard deviations are illustrated for longer training and test intervals up to $2000 \mathrm{~ms}$. C, D The firing rates of the Poisson neurons were set to $1000 \mathrm{~Hz}$. The timing and standard deviations for various noise levels by increasing the weights of the Poisson neurons are illustrated. The lighter the color, the lower the background current and the higher the noise level. The RMSE $E_{t}$ and RMSE $E_{1}$ values for these levels are shown in Table 1.

\section{Mechanisms of time perception}

Given that time perception, especially Weber's law, works within the state-dependent PFC model at least for lower noise levels and intervals up to $750 \mathrm{~ms}$, we next wanted to understand the underlying mechanisms. In a first step, we performed correlation studies to identify if there are interval encoding pools of neurons and if yes, how they differ from non-predictive neurons and from neurons which are predictive for other intervals. As a next step, we performed ablation studies to determine which components are critical for which aspect of timing in our model and conducted simulation studies and mathematical analyses on a simplified model to explain the origin of the scalar property. In order to rule out other possible mechanisms, we additionally tested for the existence of sequential activation of groups, e.g. synfire chains, and ramping activity in the model.

\section{Correlation studies}

To identify interval encoding pools, we assigned each neuron via its highest readout weight contribution to the corresponding trained interval as was already done in [11] (for details, see Methods). All weights below 0.01 were set to 0 and neurons with all weights below 0.01 were not assigned to any pool. Sorting the readout weight matrix 


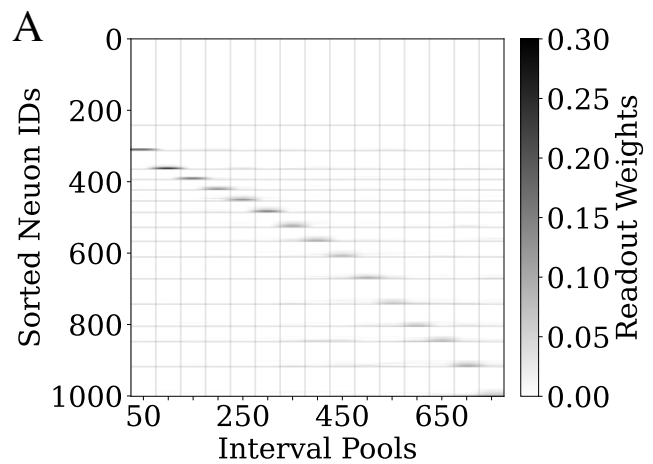

B
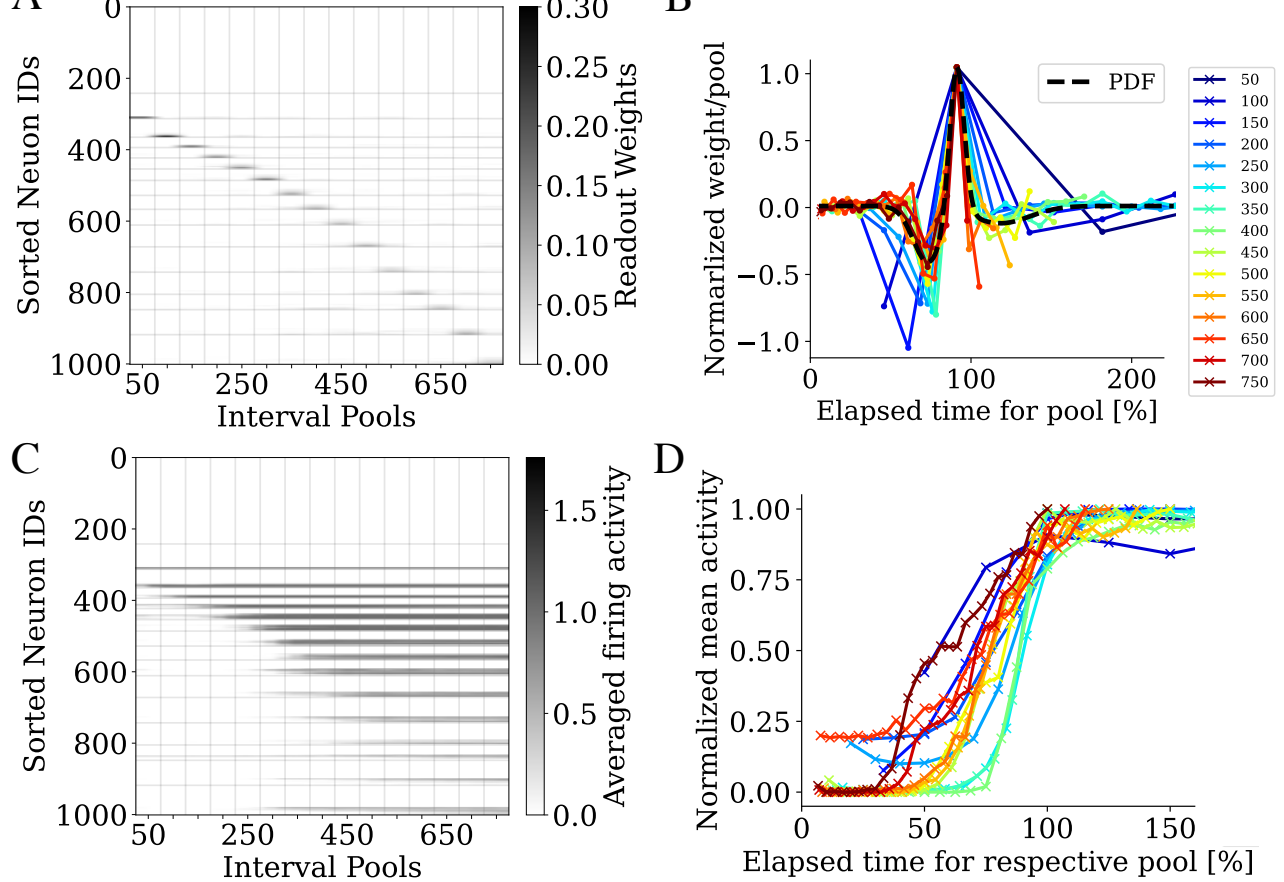

Fig 6. Readout weights and mean activity within interval encoding pools

A. The neuron IDs were sorted by their strongest weights to a readout unit such that each readout unit can be associated to neurons specific for that interval. $\mathbf{B}$ The normalized averaged weights of the neurons within a pool is plotted against the elapsed time for a respective pool for all readout intervals (color-coded) and fitted to a probability density function (dashed black line). C The averaged states over 50 trials are shown for the sorted neurons within a pool and again $\mathbf{D}$ the normalized averaged activity within a pool is calculated for each test intervals and plotted against the elapsed time for respective pool for each readout interval (color-coded).

according to the assigned per interval pools and within each pool from low to high weights yields Fig. 6A. Calculating the normalized averaged weight distribution from network to each pool before thresholding, we see stereotype temporal receptive fields, i.e. weights which peak near the time that the respective pool represents and suppress contributions from both earlier and later pools in an asymmetric fashion (Fig. 6B). Furthermore, we compared the states of these neurons in response to the second stimulus and found that the firing rate increases until $100 \%$ of the elapsed time for respective pool is reached and saturates beyond $100 \%$, see Fig. 6C, D.

Additionally, we compared the averaged membrane potential of excitatory neurons within a pool during the first stimulation at $t=1500 \mathrm{~ms}$, cf. Fig. 7A, finding a clear shift in spiking towards longer time for higher intervals. To determine if the neurons to encode smaller intervals are selected by the smaller distance of the membrane potential $5 \mathrm{~ms}$ before the first stimulation at $t=1495 \mathrm{~ms}$ to the threshold potential of that neuron, we calculated the distance of these neurons to their threshold potential at the time of the first stimulation. For the neurons which were already above threshold and for neurons which did not fire at all, we calculated the distance to the peak value within a range of $1500-1530 \mathrm{~ms}$. Doing so, a linear trend with a slope of 0.005 ( $\mathrm{p}>0.002$, $R^{2}=0.53$ ) for the excitatory neurons could be reported (one-way ANOVA: $\mathrm{F}=3.037$ 

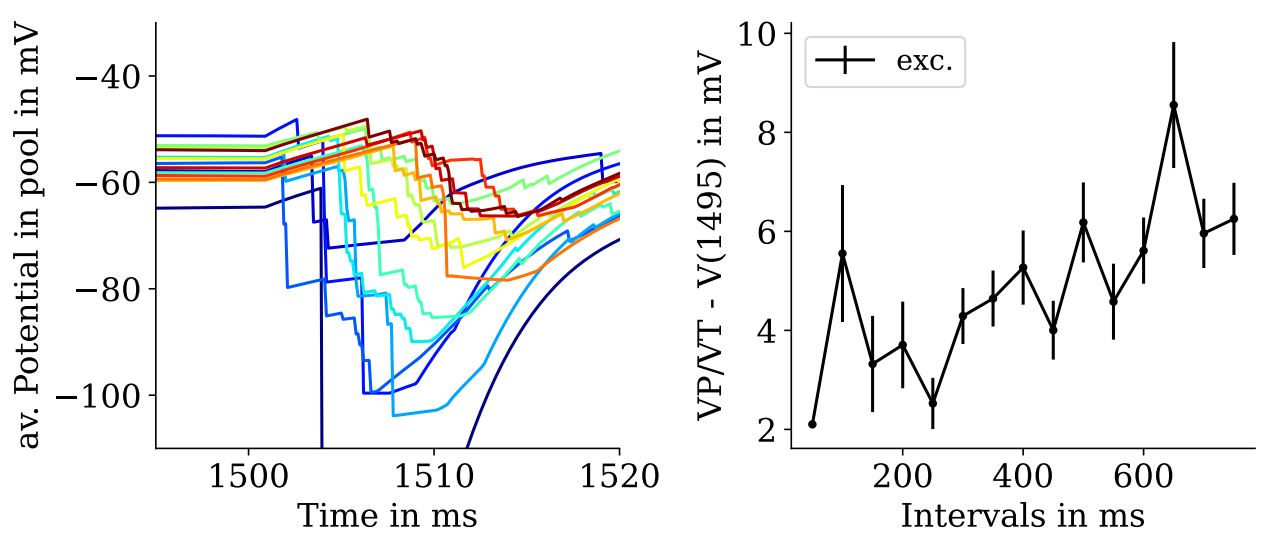

Fig 7. Pool averaged membrane potential of excitatory neurons.

A Averaged membrane potentials of excitatory neurons for each interval encoding pool are illustrated. B Difference of averaged membrane potential $5 \mathrm{~ms}$ before the first stimulation (i.e. $t=1495 \mathrm{~ms}$ ) to $V_{T}$ (or peak potential if the respective did not elicit any action potential) during the first stimulation.

and $\mathrm{p}<.001)$. Hence, selected neurons from shorter intervals are relatively closer to their respective thresholds in comparison to neurons in longer interval pools. This effect could not be seen within the neurons below the threshold witihn the pools (slope $=$ $\left.-0.002, R^{2}=0.03\right)$.

In a next step, we separately examined the currents within a pool for excitatory and inhibitory currents as shown in Fig. 8A, B. As expected all synaptic currents peak during the first stimulus and for their corresponding second stimulation. Furthermore, if we compare the absolute amplitude of synaptic currents before the first stimulus (1000 $1500 \mathrm{~ms}$ ), in response to the first or second stimuli, we find an increase in excitatory and inhibitory currents for pools encoding longer intervals in all three cases (Fig. 8C, D and E).

Table 2. Synaptic currents within interval encoding pools

\begin{tabular}{lcc} 
& one-way ANOVA & linear regression $(50-400 \mathrm{~ms})$ \\
\hline $\begin{array}{l}\text { before stimulation } \\
\text { Exc. currents }\end{array}$ & $\mathrm{F}=3.89, \mathrm{p}<.001$ & slope $=0.25, R^{2}=0.90$ \\
$\begin{array}{l}\text { Inh. currents } \\
\text { Both }\end{array}$ & $\mathrm{F}=4.50, \mathrm{p}<.001$ & slope $=-0.39, R^{2}=0.93$ \\
first stimulation & $\mathrm{F}=1.34, \mathrm{p}=0.19$ & slope $=-0.14, R^{2}=0.83$ \\
Exc. currents & $\mathrm{F}=2.43, \mathrm{p}<.01$ & slope $=1.89, R^{2}=0.87$ \\
Inh. currents & $\mathrm{F}=6.09, \mathrm{p}<.001$ & slope $=-1.19, R^{2}=0.88$ \\
Both & $\mathrm{F}=1.22, \mathrm{p}=0.26$ & slope $=1.16, R^{2}=0.33$ \\
second stimulation & & \\
Exc. currents & $\mathrm{F}=3.92, \mathrm{p}<.001$ & slope $=2.55, R^{2}=0.91$ \\
Inh. currents & $\mathrm{F}=6.84, \mathrm{p}<.001$ & slope $=-1.14, R^{2}=0.85$ \\
Both & $\mathrm{F}=1.51, \mathrm{p}=0.11$ & slope $=1.63, R^{2}=0.42$
\end{tabular}

This linear effect could especially be seen for intervals up to $400 \mathrm{~ms}$, see slopes and $R^{2}$ values in Table 2, still it was statistically verified for the whole range for excitatory and inhibitory currents separately and together, cf. one-way ANOVA in Table 2. In order to determine the excitability of the neurons within a pool, we calculated the 
A

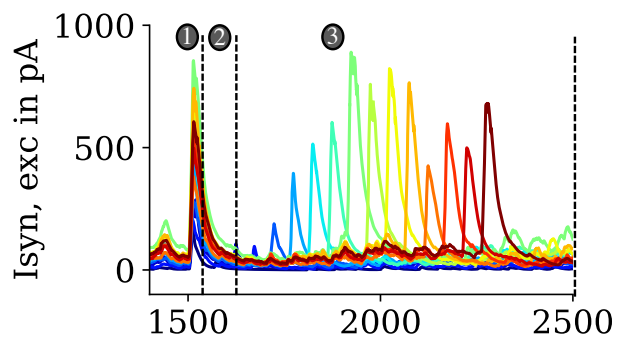

$\mathrm{C}$

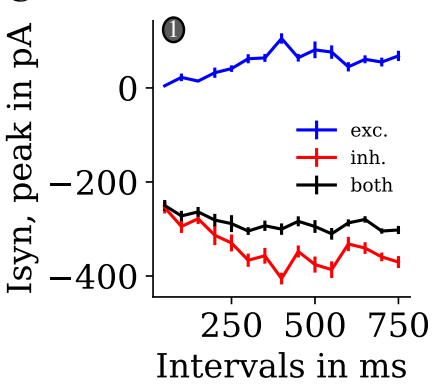

$\mathrm{D}$

\section{B}

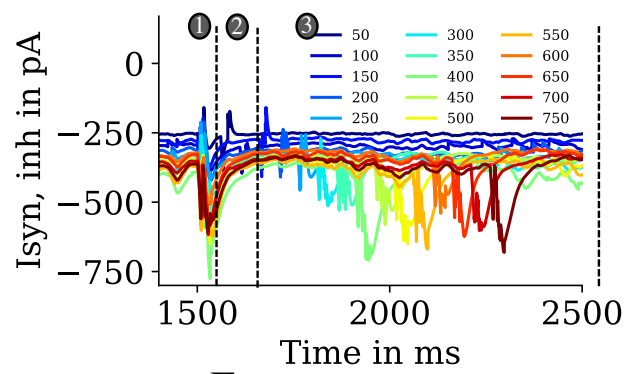

$\mathrm{E}$

Fig 8. Synaptic currents within the pools.

The averaged excitatory $\mathbf{A}$ and inhibitory $\mathbf{B}$ synaptic currents of the neurons within a pool. The three circles and the dashed lines remark the three time points at which the synaptic currents are compared, namely before stimulation (1) C, during first stimulation (2) D and during the second stimulation (3) E. For each case, the excitatory, the inhibitory and the overall peak current (positive for excitatory currents and negative for inhibitory currents) are illustrated with the standard error.

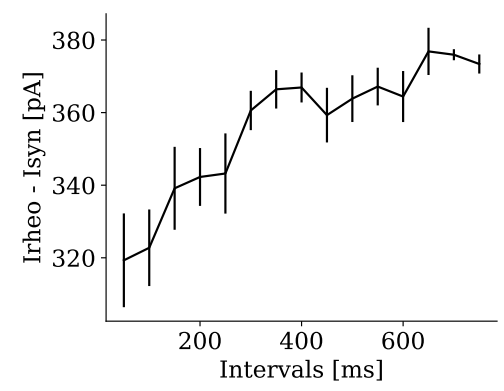

Fig 9. Excitability of interval encoding pools

The rheobase is calculated as described in section Rheobase in Materials and Methods and subtracted by the total synaptic current within the pools before stimulation, cf. black line in Fig. 8C.

averaged rheobase within a pool and subtracted this by the averaged total synaptic current within the range $1000 \mathrm{~ms}-1495 \mathrm{~ms}$, cf. black line in Fig. 8C. Although both, the rheobase and the total synaptic current did not show a linear trend, the difference of the two did increase over the whole range $50-750 \mathrm{~ms}$ (oneway ANOVA: $\mathrm{F}=5.10, \mathrm{p}$ $<.001$, slope $=0.075, R^{2}=0.82$ ), see Fig. 9, implying that a higher overall current is needed to make the neurons in pools encoding longer intervals fire. In contrast to that, if we consider only those neurons within the pools below the weight threshold of 0.01 , 
the difference of the rheobase to the synaptic current does not show a linear trend (slope $=0.065$ and $R^{2}=0.20$ ).

A
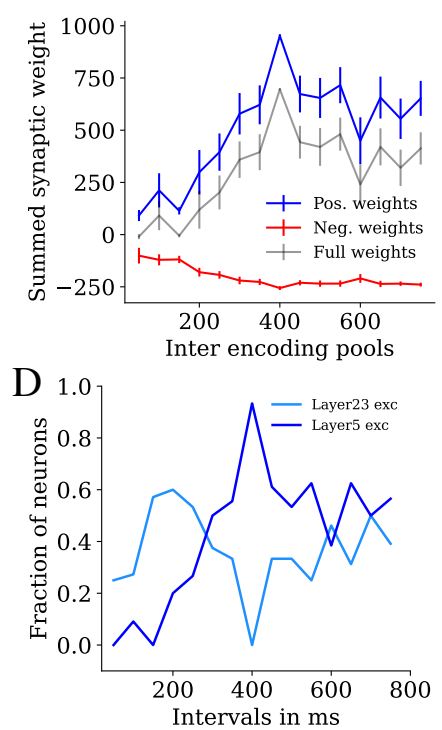

B
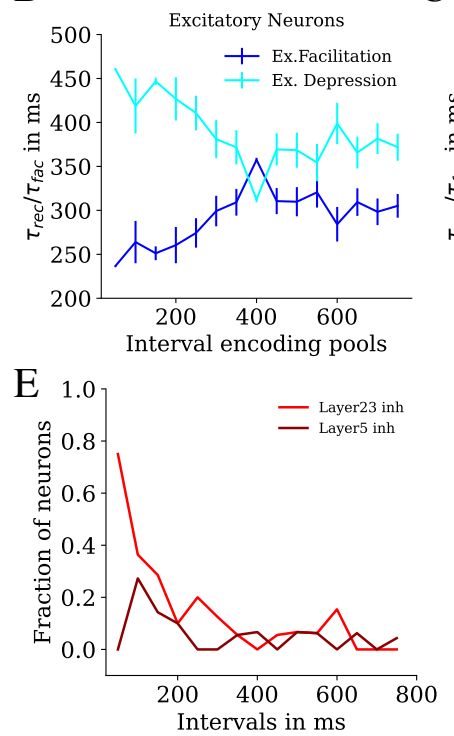

$\mathrm{C}$

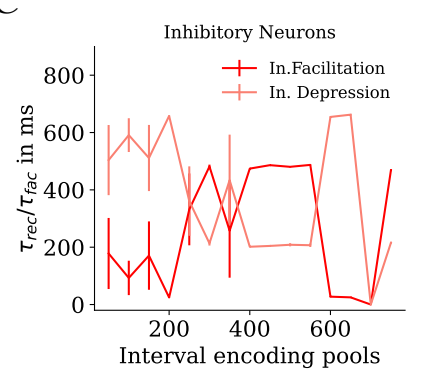

Fig 10. Neuronal and synaptic properties within interval encoding pools

A The averaged summed synaptic weight onto an interval encoding pool is presented for positive weights (blue), for negative weights (red) and together (black). The time constants of STP, $\tau_{f a c}$ and $\tau_{f a c}$ are shown for neurons within an interval encoding pool for excitatory $\mathbf{B}$ and inhibitory $\mathbf{C}$ neurons. The neuron and layer types of the neurons within a pool were checked for excitatory $\mathbf{D}$ and inhibitory $\mathbf{E}$ neurons.

So far, we analyzed the membrane potentials and the synaptic currents to understand the mechanisms of time perception. Next, we examined whether the assignment of a neuron to one of the interval-encoding pools already depends on the synaptic parameters. Indeed, the averaged synaptic weights (black: slope $=0.84, R^{2}$ $=0.70$ ), positive (blue: slope $=2.28, R^{2}=0.90$ ) and negative (red: slope $=-0.46, R^{2}=$ 0.96 ) weights, cf. Fig. 10A, as well as the facilitating time constants within the excitatory, Fig. 10B (slope $=0.30, R^{2}=0.85$ ), and inhibitory neurons, Fig. 10C (slope $=0.98, R^{2}=0.52$ ), all show an absolute increase with duration of the encoded intervals in the respective pools, up to $\sim 400 \mathrm{~ms}$. Simultaneously, the fraction of the depressing time contrasts are decreased up to $\sim 400 \mathrm{~ms}$, cf. Fig. 10B for excitatory neurons (slope $=-0.35, R^{2}=0.83$ ) and $\mathrm{C}$ for inhibitory neurons (slope $=-0.97, R^{2}=0.51$ ). Finally, we checked whether layer or neuron type might play a role for interval specific pools, cf.

Fig. 10D,E. Especially for layer $2 / 3$ neurons we find an increased fraction of excitatory and a decreased fraction of inhibitory neurons up to $\sim 400 \mathrm{~ms}$.

As the same linear trends in synaptic weights, time constants and neuron type are not pronounced for neurons within the pool below the threshold, see Suppl. Fig. 3 in Supplementary Materials, we hypothesize that these properties might be important factor for encoding time.

\section{Ablation experiments}

As all three timing properties could be reproduced within this state-dependent PFC model, we further analyzed which of the components of the model were critical for our results. There are mainly three ingredients which might be critical, namely the synaptic 
processes due to the long time constants, the heterogeneity within the data-driven
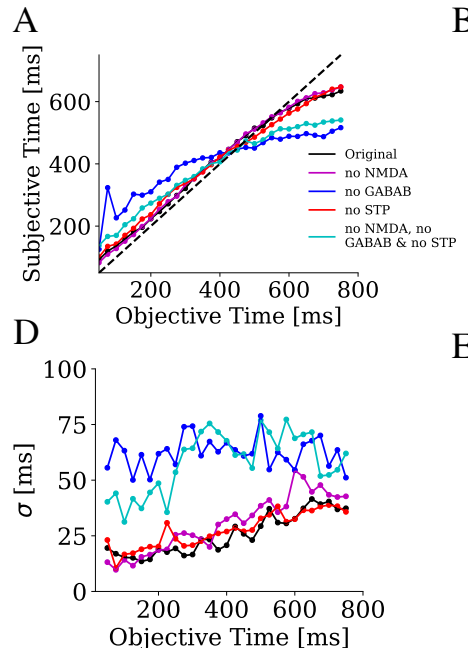

B

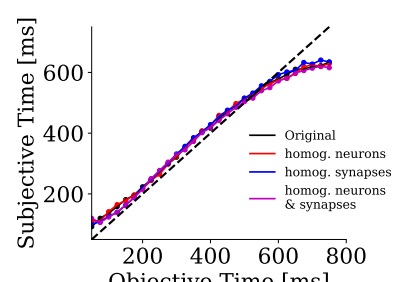

$\mathrm{E}$

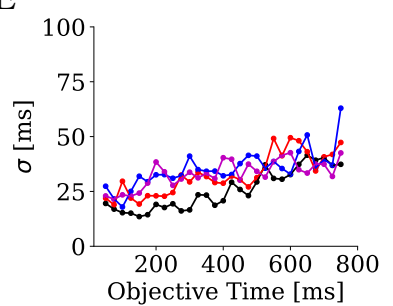

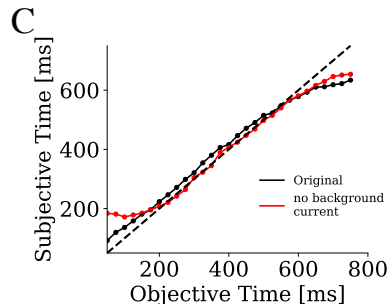

F

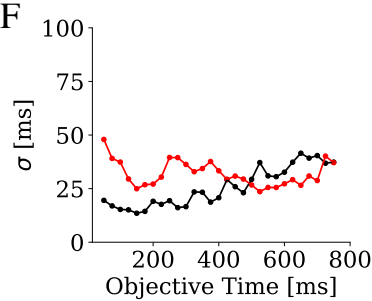

Fig 11. Ablation studies.

The estimated time and the standard deviations are illustrated for different ablation studies. A, D refers to the removal of synaptic properties, like NMDA (magenta), $\mathrm{GABA}_{\mathrm{B}}$ (blue) and STP (red). B, E The ablation of the heterogeneity of the neuronal parameters (red), of the synaptic parameters (blue) and of both parameters (magenta) is illustrated here. The additional reduction of all three STP types to one type within a subtype is shown in cyan. $\mathbf{C}, \mathbf{F}$ shows the result for removing the background current within the PFC model (red). To compare with the original case, the results from above are replotted again in black. The corresponding error values and fit parameters are shown in Table 3 and 4.

For the synaptic processes, there are three types with long time constants, which might influence the estimation of time, NMDA, GABA $\mathrm{B}$ and STP. In order to test their specific influence in time estimation, we conducted ablation experiments by removing each of these synaptic types, adjusting the background current synaptic weights to compensate for missing missing inputs and retraining the readout layer. The $\mathrm{RMSE}_{\mathrm{t}}$ values, fit parameters of Vierordt's law and the Lyapunov exponents are reported in Table 3 and the Weber's law related parameters, such like RMSE ${ }_{1}, R_{M S E}$, and fit parameters to linear and square-root fit are presented in Table 4. The Lyapunov exponents are calculated for some ablation studies to test whether the intrinsically generated noise by chaos (positive Lyapunov exponents) might be important for generating the scalar property, see Methods.

Removing NMDA synapses and STP had no effect in terms of estimated times, see Fig. $11 \mathrm{~A}$ and $\mathrm{B}$ in magenta and red, Table 3 and Table 4, although removing STP yielded a broader of firing rates. In contrast, if $\mathrm{GABA}_{\mathrm{B}}$ is removed, such that inhibition is limited to $\mathrm{GABA}_{\mathrm{A}}$ with much faster time constants, time perception is worse, leading to much higher $\mathrm{RMSE}_{\mathrm{t}}$ values and a much pronounced Vierordt's law, see Table 3 and Fig. $11 \mathrm{~A}$ in blue. Furthermore, both RMSE $\mathrm{F}_{\mathrm{l}}$ and $\mathrm{RMSE}_{\mathrm{s}}$ show similar values of $\sim 7 \mathrm{~ms}$, see Table 4 , such that it is unclear if time perception follows Webers law. To test the combined synaptic effect of these three types, we ablated all of these in a further simulation, which yielded a better $\mathrm{RMSE}_{\mathrm{t}}$ than without $\mathrm{GABA}_{\mathrm{B}}$, but still, a worse $\mathrm{RMSE}_{\mathrm{l}}$ and $\mathrm{RMSE}_{\mathrm{s}}$.

As a next step, we wanted to test the role of the heterogeneity in neuron and 
Table 3. Ablation studies to identify the components of linear timing

\begin{tabular}{lcccc} 
Ablation & $\mathrm{RMSE}_{\mathrm{t}}[\mathrm{ms}]$ & Slopes & $\mathrm{IP}_{\mathrm{avg}}[\mathrm{ms}]$ & Lyapunov \\
\hline \hline original & $49.3 \pm 10.5$ & $0.83 \pm 0.05$ & 520.0 & 7.0 \\
& & & & \\
$\begin{array}{l}\text { Synaptic mechanisms } \\
\text { no NMDA }\end{array}$ & $48.0 \pm 13.7$ & $0.85 \pm 0.06$ & 563.7 & \\
no GABA & $140.6 \pm 18.2$ & $0.42 \pm 0.09$ & 447.0 & 151.0 \\
$\begin{array}{l}\text { no STP } \\
\text { no NMDA, no GABA } \mathrm{B} \&\end{array}$ & $53.3 \pm 12.32$ & $0.79 \pm 0.06$ & 469.2 & \\
no STP & $106.3 \pm 18.1$ & $0.59 \pm 0.08$ & 444.2 & \\
& & & & \\
$\begin{array}{l}\text { Heterogeneity } \\
\text { homogen. neurons }\end{array}$ & $50.9 \pm 15.4$ & $0.86 \pm 0.05$ & 570.7 & \\
homogen. synapses & $54.2 \pm 13.2$ & $0.82 \pm 0.05$ & 546.6 & \\
homogen. neurons, synapses & $54.2 \pm 16.4$ & $0.82 \pm 0.06$ & 515.3 & \\
& & & & \\
$\begin{array}{l}\text { Trial to trial variability } \\
\text { no background current }\end{array}$ & $55.5 \pm 10.6$ & $0.81 \pm 0.05$ & 220.9 & -15.9
\end{tabular}

synapse parameters, which are drawn from various distributions [41], by using homogeneous parameters within each neuron type. To this end, we first removed the heterogeneity within each neuron type for the neuronal parameters, as well as for the synaptic parameters and lastly for both parameter sets. For all three cases, $\mathrm{RMSE}_{\mathrm{t}}$, see Table 3, and $\mathrm{RMSE}_{1}$, see Table 3, is increased compared to the original case. Using homogeneous parameters for neuronal and synaptic parameters resulted in a slight shift of the standard deviations closer to the square-root function with $\mathrm{RMSE}_{\mathrm{s}}=3.9 \mathrm{~ms}$, see Fig. 11D in magenta. As the current model includes three different types of STP selected probabilistically, we additionally reduced the types to only one type per neuron type in a layer and selected the type with highest probability. By doing so, time perception is disrupted with much higher error of $\mathrm{RMSE}_{\mathrm{t}}>120 \mathrm{~ms}$ and a larger error to linear and square-root fit is found, cf. cyan line in Fig. 11D.

The trial to trial variability is generated either by internal dynamics, e.g. chaos, or external noise. In order to determine if the activation of interneurons in between two subsequent stimulations, a so called interneuron bridging, might be responsible for the linear timing results, we removed the background current to all neurons, such that no pronounced activity during intervals could be seen. Removing the background current yielded an intact timing, but a disrupted Weber's law with a negative slope of the fit of -0.01 and missing chaos indicated by the negative Lyapunov exponent, compare with Table 3 and Fig. 11F. Furthermore, we showed before that increasing noise levels up to $1000 \mathrm{~Hz}$ yielded larger errors and gradually shifts the standard deviation away from Weber's law.

\section{Origin of the scalar property}

Here, we discuss how the scalar property of the timing errors arises in the state-dependent model. First, we provide an overview over the proposed mechanism:

1. The network is divided in pools with similar neuronal and synaptic properties among its members. All pools are activated by the first stimulus and are hindered from firing again by a strong inhibitory currents, which is kept active long after the first stimulus by the slow $\mathrm{GABA}_{\mathrm{B}}$ component. Due to the considerable 
Table 4. Ablation studies to identify the components of Weber's law

\begin{tabular}{|c|c|c|c|c|}
\hline Ablation & $\begin{array}{c}\mathrm{RMSE}_{1} \\
{[\mathrm{~ms}]}\end{array}$ & $\begin{array}{l}\text { linear fit } \\
\text { params. }\end{array}$ & $\begin{array}{l}\mathrm{RMSE}_{\mathrm{s}} \\
{[\mathrm{ms}]}\end{array}$ & $\begin{array}{l}\text { square-root } \\
\text { fit params. }\end{array}$ \\
\hline original & 3.5 & $0.04 x+9.8$ & 4.3 & $\sqrt{1.86 x}-0.52$ \\
\hline \multicolumn{5}{|l|}{ Synaptic mechanisms } \\
\hline no NMDA & 4.2 & $0.06 x+7.82$ & 4.4 & $\sqrt{4.15 x}-8.62$ \\
\hline no $\mathrm{GABA}_{\mathrm{B}}$ & 7.0 & $0.0 x+61.8$ & 6.9 & $\sqrt{0.01 x}-60.3$ \\
\hline no STP & 3.1 & $0.03 x+14.3$ & 3.4 & $\sqrt{1.4 x}-4.9$ \\
\hline $\begin{array}{l}\text { no NMDA, no } \mathrm{GABA}_{\mathrm{B}} \& \\
\text { no STP }\end{array}$ & 10.7 & $0.04 x+43.3$ & 10.0 & $\sqrt{2.3 x}-29.4$ \\
\hline \multicolumn{5}{|l|}{ Heterogeneity } \\
\hline homogen. neurons & 5.5 & $0.03 x+23.5$ & 5.5 & $\sqrt{1.2 x}-14.8$ \\
\hline homogen. synapses & 4.7 & $0.04 x+17.6$ & 5.0 & $\sqrt{1.7 x}-7.3$ \\
\hline homogen. neurons, synapses & 4.2 & $0.02 x+17.7$ & 3.9 & $\sqrt{0.64 x}-17.7$ \\
\hline $\begin{array}{l}\text { Trial to trial variability } \\
\text { no background current }\end{array}$ & 5.5 & $-0.01 x+35.5$ & 5.8 & $\sqrt{0.0 x}-31.6$ \\
\hline
\end{tabular}

heterogeneity of these parameters within the network, the different pools $i$ recover from inhibition at different times, reflected in a firing probability $p_{i}(t)$ that peaks close to one at a specific time $T_{i}$. The time course of $p_{i}$ scales similarly for all pools, e.g. the respective curves $p_{i}\left(t / T_{i}\right)$ are almost identical for the different pools if time is scaled by $T_{i}$.

2. Each pool consists of $N_{i}$ neurons, each with firing probability $p_{i}(t)$ at a given time $t$. If each neuron is assumed to fire not more than one spike in response to the second stimulus (which is true for the vast majority of neurons within the pools), the mean and standard deviation of the firing rate $F R_{i}$ (i.e., number of firing neurons) at the time of the second stimulus is given by the binominal distribution.

3. The firing rates of the different pools are than translated into tuning curves $O_{j}$ of the different output neurons $j$ by convolving them with a kernel of synaptic weights that also takes the form of a relatively homogeneous temporal receptive field. Thus, all output neuron activities $O_{j}$ show a similar relation between their mean and their standard deviation, just as in the firing rates $F R_{i}$ of the pools in the network.

4. Finally, the activity $O_{j}$ of all output neurons is multiplied with the interval duration each output neuron represents and summed up to form the estimate of the interval duration between the two stimuli. As only a few output neurons with similar represented durations $T_{j}$ contribute to this estimate, the standard deviation of the estimate scales approximately linear with its mean, which is the scalar property.

First, recall that the binominal distribution describes the number of successes (here: spikes) in a sequence of $N$ random experiments (here: neurons), where each success has the same probability $p$. Thus, the binominal distribution can be used to estimate the firing rate in a pool with $N_{i}$ neurons and $p_{i}(t)$ at time $t$ (we will abbreviate $p_{i}(t)$ with $p_{i}$ in the following when the particular time $t$ does not need to be emphasized). In particular, the mean firing rate $\left\langle F R_{i}\right\rangle=N_{i} p_{i}$ and its standard deviation 
$\sigma_{F R_{i}}=\sqrt{N_{i} p_{i}\left(1-p_{i}\right)}=\sqrt{N_{i}\left(p_{i}-p_{i}^{2}\right)}$ are known from this distribution. For small to moderate $p_{i}, \sigma_{F R_{i}}$ can be approximated by $\sqrt{N_{i} p_{i}}$, neglecting $p_{i}^{2}$.

For clarity, we elaborate on each of the above steps in reverse order. Regarding step 4, Fig. 12A shows the relation between the mean and the standard deviation of the activity of each of the output neurons $O_{j}$ (cf. lines and shaded areas in Fig. 2A). This relation can be well fitted to the function $\sigma_{O_{j}}=\sqrt{c_{i}<O_{j}>}$ with a relatively constant $c_{i}$ across pools. As the duration estimate $T_{\text {est }}(t)$ is formed by summing over the activities $O_{j}$ of each output neurons multiplied by the duration $T_{j}$ that neuron represents, mean and standard deviation of the estimate are given by $<T_{\text {est }}(t)>=\sum_{j} T_{j}<O_{j}>$ and $\sigma_{T_{\text {est }}(t)}=\sqrt{\sum_{j} T_{j}^{2} \sigma_{O_{j}}^{2}}$, respectively.

In the following, we evaluate both quantities at one of the represented times $t=T_{i}$ for simplicity. Under the assumption that all tuning curves $\left\langle O_{j}\right\rangle$ have the same shape, e.g. mean-shifted Gaussians, we can interpret the values $\left\langle O_{j}\left(T_{i}\right)>\right.$ of the different output functions $j$ at this time $T_{i}$ as that values of the single function $T_{i}$ at different times $T_{j}$. Given the fact that the output activities are normalized such that $\sum_{j}<O_{j}(t)>=1$, we can interpret $\left\langle O_{i}>\right.$ as the probability distribution of the represented times $T_{j}$. Thus,

$$
<T_{\text {est }}\left(T_{i}\right)>=\sum_{j} T_{j}<O_{i}\left(T_{j}\right)>=<T>=T_{i}
$$

and, using $\sigma_{O_{j}} \approx \sqrt{c<O_{j}>}$ and $\operatorname{Var}\left(T_{i}\right)=<T_{i}^{2}>-<T_{i}>^{2}$,

$$
\sigma_{T_{\text {est }}\left(T_{i}\right)}=\sqrt{\sum_{j} T_{j}^{2} \sigma_{O_{j}}^{2}}=\sqrt{c \sum_{j} T_{j}^{2} O_{i}\left(T_{j}\right)}=\sqrt{c} \sqrt{<T_{i}^{2}>\sqrt{c}} \sqrt{\operatorname{Var}\left(T_{i}\right)+<T_{i}>^{2}} .
$$

Together, eqn. 1 and 2 yield the generalized Weber law

$$
\sigma_{T_{\text {est }}\left(T_{i}\right)}=\sqrt{c} \sqrt{\operatorname{Var}\left(T_{i}\right)+<T_{\text {est }}\left(T_{i}\right)>^{2}}
$$

with constant $\operatorname{Var}\left(T_{i}\right)$ that depends on the spacing of the represented durations $T_{j}$ (each $50 \mathrm{~ms}$ in our case) and the Weber fraction $\sqrt{c}$. Indeed, the Weber fractions calcuated from the duration estimates and the square root of the sum of the variances in all output neurons $O_{j}$ are in good agreement to each other, unless for small durations below $200 \mathrm{~ms}$ (Fig. 12B), where the constant value becomes dominant.

Regarding step 3, we have seen in Fig. 6B and D that both firing rates and readout weights follow highly stereotype temporal profile for each pool $i$ when time is scaled to the duration $T_{i}$ that is represented in that pool. The firing rates (of the firing probabilities $p_{i}$, more precisely) can be well described by a cumulative normal distribution (Fig. 12C, where $p_{i}$ is plotted for a given time $t$ as a function of the represented time $T_{i}$ for all pools) and the weights follow an asymmetric version of the Mexican hat function (Fig. 6B, black line). The symmetric Mexican hat is a well-known synaptic profile in spatial processing in the visual system [51, 76]. Here, the asymmetric version accounts for the different slopes of firing rate distribution before and after the interval that is represented in the corresponding output neuron (Fig. 12C).

Regarding step 2, Fig. 12D shows the relation between mean and standard deviation of the firing probabilities $p_{i}$ of each pool. $p_{i}$ is estimated by normalizing the mean firing rates $F R_{i}$ to the maximal value in each pool. The standard deviation is fitted to the relation from the binominal distribution $\sigma_{F R_{i}}=\sqrt{N_{i} p_{i}\left(1-p_{i}\right)}$ and the values in the figure are divided by the fit parameter $\sqrt{N_{i}}$. The fit is excellent for pools representing short intervals, but gets worse for $T_{i}>400 \mathrm{~ms}$. The estimated $N_{i}$ values from the fit qualitatively match the actual pool sizes [statistics]. 
A
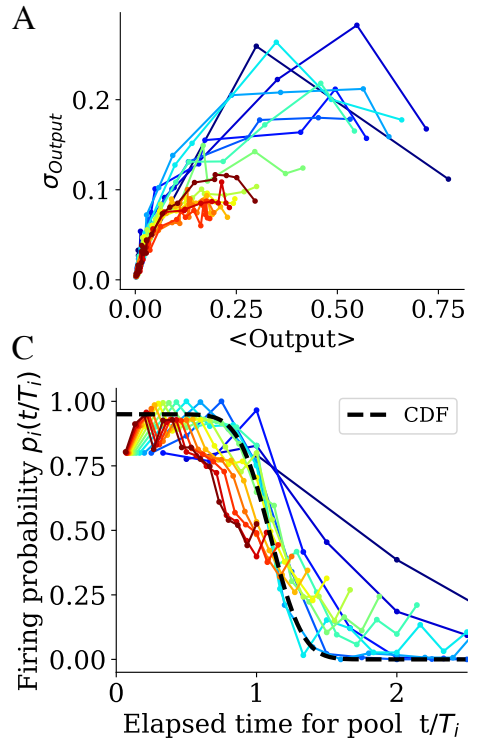

B
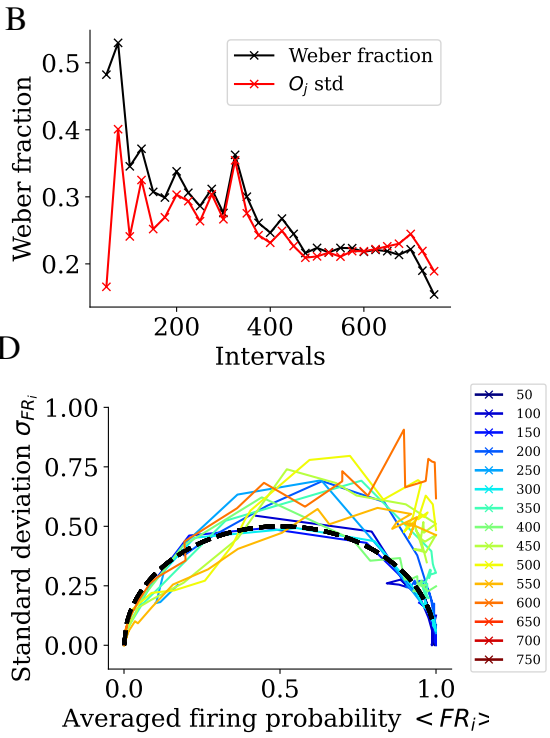

Fig 12. Origin of the scalar property

A. Standard deviations of the output neuron activity as a function of their mean activity. See panel D for the color code. B. Weber fractions (black curve, standard deviation of the duration estimated divided by the mean) and square root of the sum of the variances of all output neurons (red curve) for each of the encoded interval durations. C. Firing probability of each pool $i$ as a function of time, scaled by the duration $T_{i}$ that is represented in that pool. See panel $\mathrm{D}$ for the color code. The dotted line shows the best fit of a normal cumulative distribution function (CDF) to all curves. D Standard deviation of the firing probabilities as a function of the mean shown in panel C. Apart from pools encoding long durations, the curves are well fitted by the relation predicted by the binominal distribution (dotted line). The colors correspond to the intervals that are represented in each pool (see color bar, cf. Fig. 2A).

Regarding step 1, the cumulative normal distribution of the firing rates (Fig. 12C) follows from the fact that each neuron fires as a soon as its particular firing threshold is crossed. Given that the membrane potential of each neuron is driven by noise that can be well approximated by a normal distribution, the probability to cross the threshold at a given time is the cumulative normal distribution with the difference of the mean membrane potential $\left\langle V_{i}(t)\right\rangle$ from the threshold $V_{t h, i}$ as the mean. The standard deviation of the membrane potential does not systematically change over time, as neurons are driven back toward their resting potential by leak currents, which can be approximated by the Ornstein-Uhlenbeck process [81] for which the standard deviation is constant in time. Thus, the change in $p_{i}(t)$ over time is mainly driven by changes in the mean membrane potential $\left\langle V_{i}(t)\right\rangle$. The main driver of this change over time is the slow $\mathrm{GABA}_{\mathrm{B}}$ inhibition after the first stimulus, which effectively prevents neurons from firing in response to a second stimulus within a certain window of time. Apart from the time constant of the $\mathrm{GABA}_{\mathrm{B}}$ conductance, this time window is determined by the neuronal and synaptic parameters within each pool. As we have seen in Fig. 9, the mean current difference to the neurons' rheobase increases for pools encoding longer intervals (which can be translated into the difference between the average membrane potential and the firing threshold). Thus, more of the inhibiton from the first stimulus must be wear off before neurons those pools can respond to the second stimulus, so that happens at later times. In summary, the increase of $p_{i}$ over time is mainly due to a 
gradual decay of long-term inhibition which decreases the difference between average membrane potential and firing threshold and thus, increases the chance of each neuron to fire. The different parameters in each pool determine the speed by which $p_{i}$ increases over time. To reconstruct the scaling of $p_{i}$, we have constructed a minimal model simulating a single neuron that receives a large amount of random, balanced excitatory and inhibitory currents and is subject to a $\mathrm{GABA}_{\mathrm{B}}$ current following the time of the first stimulus and to a fixed depolarization at the time of the second stimulus.

Simulating this neuron of a large number of trials allows to estimate $p_{i}$ for a given firing threshold $V_{t h, i}$. For simplicity, we simulate the different pools by varying $V_{t h, i}$, although the above results suggest a variation of the synaptic properties. For each simulated pool $i$, we compute the first time $T_{i}$ at with $p_{i}$ exceeds $95 \%$ and record $T_{i}$ as the represented duration of this pool. When time is scaled by this duration $p_{i}\left(t / T_{i}\right)$ shows the same cumulative normal distribution time course for each pool (Fig. 13), as we see in the network model (Fig. 6D).

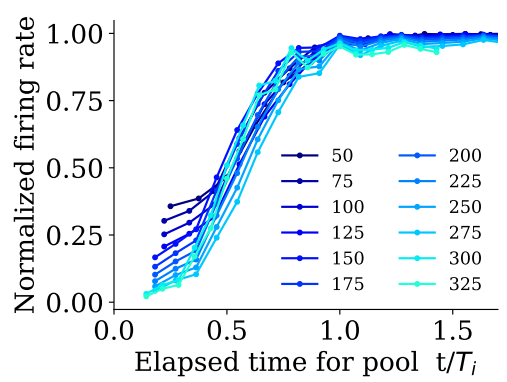

Fig 13. Results from the minimal model.

Each of the curves was generated by a different threshold for the membrane potential to elicit a spike. Higher thresholds lead to longer durations that need to elapse before that first spike (cf. Fig. 9). When scaling this time by the duration $T_{i}$ at which the neuron spikes with 95\%, the firing rate curves for all durations largely overlap, as in Fig. 6D for the full model.

\section{Deviations from the scalar property and the origin on Vierordt's law}

The theory of the scalar property derived above rests on a number of assumptions. First, firing rate curves and weight kernels need to be roughly the same for all pools after scaling time with the duration each pools represents (Fig. 6B and D). Second, output activations must be small enough such that the standard deviation can be approximated by the square root of the mean (Fig. 12A). And finally, the output tuning curves need to have the same shape for all output neurons. Here, we discuss consequences by violating the third assumption. As apparent from Fig. 2A, the output curves show the same shape up to $350 \mathrm{~ms}$, but quickly grow broader for longer intervals, violating the third assumption. In the most extreme case, where all curves $O_{j}$ are completely flat such that each intervals contributes with the same value, it follows from the normalization of $O_{j}(t)$ to one that for each time, the same duration estimate $\left\langle T_{j}\right\rangle$ is generated, which is the average over all estimated intervals $T_{j}$. Likewise, that standard deviations are also constant in time in this case. In intermediate cases, combining constant, sharp tuning curves $O_{j}$ for short intervals $T_{j}$ and increasingly broader curves for longer intervals, the scaling of both the mean and standard deviation are shifted away from the linear relations described in Eq. 1 and 3 and towards constants values. This implies that deviations from the scalar property and Vierordt's law, the observation that long intervals are underestimated and short ones are overestimated, are mechanistically 
linked. Indeed, for most manipulations described above, a positive linear relation can be found between the slope of Vierordt's law (one meaning a one-to-one relation, zero a constant estimate for each duration) and the amount of evidence for the scalar property, quantified as the difference of the RMSE for the square root and the linear relation the more positive the difference, the stronger the evidence for the scalar property $(r=0.60, t(12)=2.60, p=0.014)$. The only exception is the ablation of $\mathrm{GABA}_{\mathrm{B}}$, which strongly decreases the slope of Vierordt's law, but yields a scaling of the timing errors that is undecided between a square root and a linear relation. Furthermore, a strong negative linear relation is found between the slope of Vierordt's law and the minimal standard deviation of the output curves $O_{j}(r=-0.96, t(12)=-11.88, p<0.01)$.

\section{Ruling out alternative mechanisms}
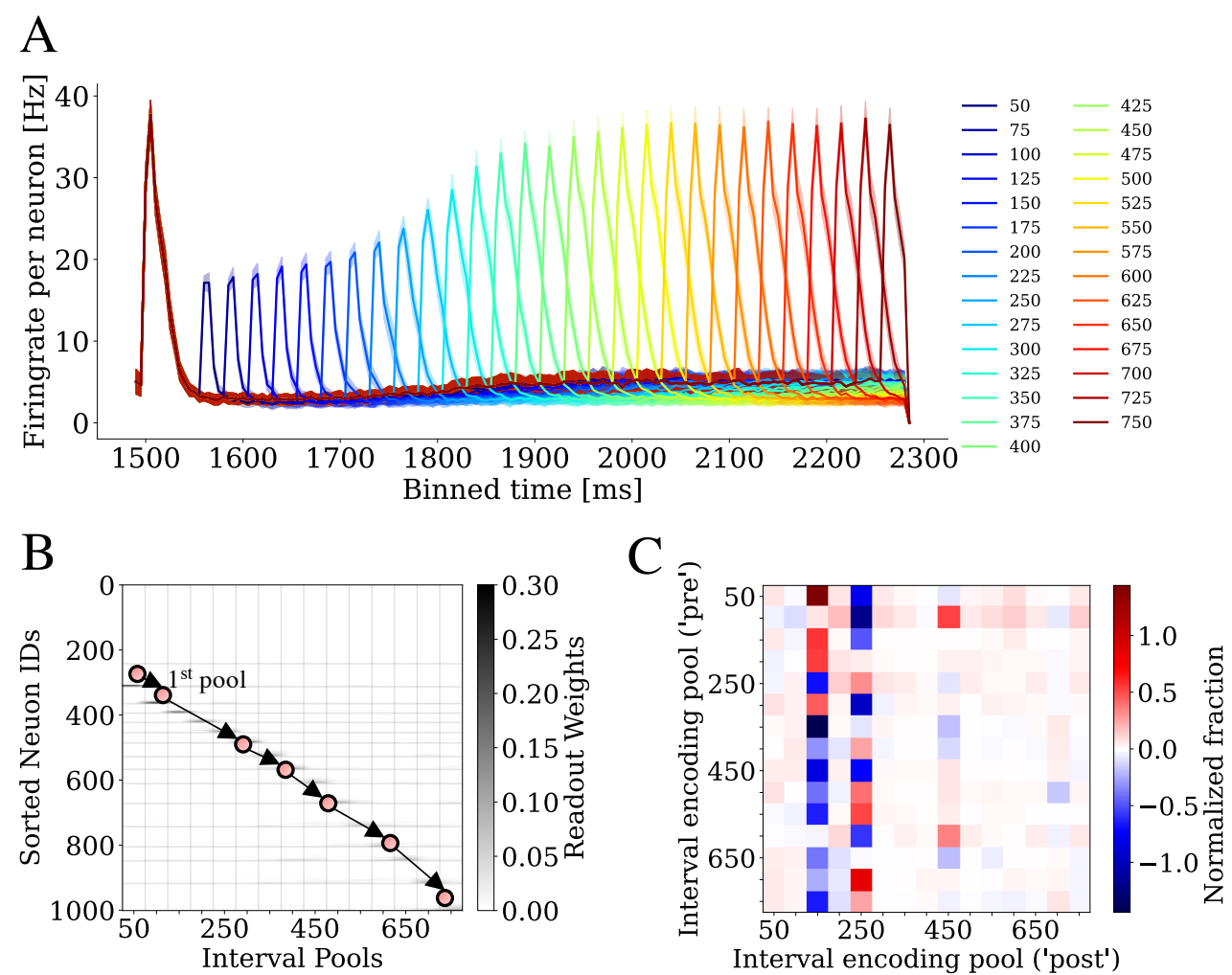

Fig 14. Ruling out alternative mechanisms.

A. The averaged binned firing rate $(10 \mathrm{~ms})$ over the test trials is plotted for each neuron to test for ramping activity. The different colors indicates the test intervals and the shaded region the standard deviation over 50 trials. B. Illustration of a hypothetical sequential activation of interval encoding pools, which is tested in $\mathbf{C}$. The synaptic weights from a pre synaptic pool to a postsynaptic pool are calculated for each pool combination and normalized by the overall input to a pool.

After finding a possible mechanism which could explain our timing results, we next wanted to rule out other possible timing mechanisms within this model. On that account, we tested whether the neurons encoding a specific pool show a ramping activity over time, cf. Fig. 14A to rule out the timing model proposed by Durstewitz [20]. We calculated the trial averaged firing rate of each neuron step wise in $10 \mathrm{~ms}$ bins for all test intervals (color-coded). An increase of the firing rate over time, which 
maximizes at the corresponding timing interval could not be seen here, see Fig. 14A.

Lastly, we tested whether there is sequential activation of subsequent pools analogous to a synfire chain, as shown in the illustration in Fig 14B. As the normalized fraction of one pool to another pool is relatively small compared to the overall synaptic weight onto the neurons (Fig. 14C) and specifically no sequential activation of pools could be seen in the pool-to-cool connectivity matrix in Fig. 14C, we can discard this idea.

\section{Discussion}

\section{Summary of main results}

The notion of time perception is well accessible through experiments in animals and humans and has allowed for the characterization of three central timing properties, namely psychophysical law [23], Webers law [29] and dopaminergic modulation [9]. So far, however, the underlying mechanisms are still unclear [68]. In this work, we adapted an existing computational model, the state-dependent model, to match a realistic PFC model and tested against the above mentioned timing properties. Specifically, 1000 simplified adaptive exponential integrate-and fire neurons were stimulated in the beginning and in the end of a range of different time intervals. Then, states of neurons were extracted based on which a readout layer was trained using least squares to predict the respective inter stimulus interval. Our model can successfully reproduce the psychophysical law and the Webers law for intervals in the range of $50-750 \mathrm{~ms}$. The dopaminergic modulation was tested by modifying neuronal and synaptic parameters and yielded within a smaller range of 200 - $600 \mathrm{~ms}$ the expected slowing down of the internal clock for an antagonistic modulation (up to -50\%) and similarly the speeding up of the internal clock for an agonistic modulation (up to $20 \%$ ).

\section{Discussion of mechanisms and limitations}

Within the model proposed here, we were able to reproduce Webers law up to $750 \mathrm{~ms}$, whereas previous computational models only reported sublinear increase of the standard deviations or not at all [37]. As the information-theoretical framework proposed by Hass and Herrmann [39] suggested that the scalar property will only be captured, if the model relies entirely on a time-dependent variance and not on a time-dependent mean or a time-dependent covariance, we were not expecting to find a linear fit of Webers law. For this reason, we aimed to find a mechanistic explanation for the scalar property in the present model that may go beyond the framework by Hass and Herrmann [39].

When elaborating on the underlying mechanisms, we found the balance between excitation and inhibition mainly driven by $\mathrm{GABA}_{\mathrm{B}}$ as the key property related to timing. The earlier the second stimulus was applied, the higher was the inhibition onto all neurons and therefore, the peak of the synaptic current was reduced. Therefore, for shorter intervals only those neurons become active which receive relatively less inhibition. Further analysis revealed that neither the heterogeneity within the model nor a single parameter is sufficient to predict a neurons assignment to an interval encoding pool. Instead, the interplay of the number of connections onto a neuron, fraction of facilitating versus depressing time constants, the excitability of a neuron and the difference of the membrane potential to a neuron's threshold shortly before the stimulation determines the assignment of a neuron to an interval encoding pool.

We also found a plausible explanation for the origin of Weber's law, combining profiles of firing probability that are scaled by the duration represented in each time-specific pool of neurons with a stereotyped temporal receptive field occurring from optimizing the readout from each of the pools in downstream units, both resulting in 
largely uniform shapes of temporal tuning curves in these output neurons and finally, constant Weber fractions. All these components work together in the way summarized above for durations up to $\sim 400 \mathrm{~ms}$, beyond which the time constants of the underlying synaptic currents $\left(\mathrm{GABA}_{\mathrm{B}}\right.$ and short-term plasticity) are exceeded and both time perception and Weber's law begin to degrade.

A major limitation within our model is that the modulation by dopamine only worked in a smaller sub region and for lower percentages of modulation. An agonistic modulation increases the overall excitability of the network by reducing the peak membrane potential, threshold potential $V_{T}$, and increasing the peak conductance of NMDA. Since excitability is the key feature within our model, this increase leads to a faster activation of the neurons of later pools shifting time to larger estimates, cf.the outputs of the $400 \mathrm{~ms}$ encoding readout unit in Fig. ?? in Suppl. Fig. 4 in Supplemental Materials. As the model is only trained up to $750 \mathrm{~ms}$ and the neurons were not trained for even larger intervals, the altered excitability for higher intervals could not be captured by any pools. As a result, the model collapses to a fixed estimated time for all intervals upon higher DA modulation. Similarly, the antagonistic modulation decreases the excitability and activates for higher time intervals earlier pools thereby slowing down the internal clock. The interval encoding pools for short intervals then collapse to another fixed interval, as the model was not trained for shorter intervals.

\section{Comparison with the original state-dependent model}

If we compare our state-dependent PFC model with the original state-dependent [11], the author showed, using 500 neurons, normalized output peaks up to $250 \mathrm{~ms}$ using $50 \mathrm{~ms}$ steps for training and peaks up to $400 \mathrm{~ms}$ using $100 \mathrm{~ms}$ steps. Our time constants for $\mathrm{GABA}_{\mathrm{B}}$ were similar, as they had the same rise time with $\tau_{\text {rise }}=100 \mathrm{~ms}$, but a smaller decay time with $\tau_{\text {decay }}=166 \mathrm{~ms}$ (here: $\tau_{\text {decay }}=200 \mathrm{~ms}$ ). Within the range 50 $250 \mathrm{~ms}$, we were also able to show similar output patterns, but for longer intervals our output peaks become more broaden. Still, we were able to perform discrimination tasks up to up to $750 \mathrm{~ms}$. The co-activation of other readout units for longer intervals could also be a biologically possible mechanism to encode time, as there is no identity-shaped time estimation in experiments, rather than an overestimation of shorter intervals and an underestimation of longer intervals, i.e. the Vierordt's law.

Within the original state-dependent model, Buonomano showed reduced timing performance when $\mathrm{GABA}_{\mathrm{B}}$ or STP are removed. We were able to confirm the importance of $\mathrm{GABA}_{\mathrm{B}}$, but in our results we find a functioning time estimation when STP is completely removed. Furthermore, he claimed that temporal tuning was specifically achieved by the variability of the synaptic weights as having zero-varianced synaptic weights did not resulted in interval discrimination. In contrast, when we performed simulations with non-heterogeneous neuronal and synaptic parameters within each combination type (differences between combinations, but equal within a combination), timing could still be encoded. Rather than the synaptic weights, the connectivity might play an even more important role for our timing tasks. Neurons which receive less negative weights from inhibitory neurons, can easily be reactivated for a second interval, than neurons receiving higher inhibitory inputs.

In summary, we claim that in addition to $\mathrm{GABA}_{\mathrm{B}}$, all parameters which determine the excitability of a neuron, e.g. membrane threshold, time constants, synaptic weights, are necessary to assign a neuron into a specific pool. Even if one type, as for example the STP is removed, there might be other parameters within the network which help to differentiate the neurons to obtain interval discrimination, given sufficient heterogeneity. 


\section{Outlook}

Computational models provide means to better understand the underlying mechanisms of timing, given that experimental properties can be reproduced. For many computational timing models, the linear timing could already be observed [39]. So far, none of these models were able to show the scalar property [37], although the scalar property was seen as the most important hallmark of time perception [39,66]. While the state-dependent PFC model is capable of reproducing Webers law, there could be alternative models capturing timing properties that we did not consider in this work.

Among a wide range of existing timing models, three more mechanisms might be promising [37], namely ramping activity, synfire chains and striatal beat model. Timing is encoded within the ramping activity by slowly increasing firing rates as a result of calcium influx which peaks when $100 \%$ of the interval has elapsed [20, 73]. Synfire chains describe a feedforward activation of subsequent pools of neurons, such that timing can be encoded by means of activity of the respective pools $[12,40]$. Within the striatal beat model, timing is encoded via neuronal oscillators with different frequency bands, meaning that groups of neurons are synchronized via synaptic interactions forming a so-called "beat" [60]. Similar to the original implementation of state-dependent model, above discussed models showed a sublinear increase of the Weber's law [40]. Therefore, we consider testing these models within the PFC model as essential future work.

\section{Materials and Methods}

\section{Network model}

To test timing within a realistic prefrontal cortex model, we used the spiking neural network model proposed by [41]. This model can reproduce key features of in vivo activity, as the neuronal parameters and synaptic parameters were constrained by anatomical and electrophysiological data from in vivo and in vitro experiments on rodents. The spiking neural network consists of two laminar components, layer $2 / 3$ and layer 5 , of which $47.0 \%$ within layer $2 / 3$ and $38.0 \%$ within layer 5 were excitatory pyramidal neurons and $10.4 \%$ within layer $2 / 3$ and the $4.6 \%$ within layer 5 were interneurons. The interneurons comprise fast spiking cells, bitufted cells, Martinotti cells and basket cells [41]. The underlying neuron model used here was the simplified adaptive exponential integrate-and-fire neuron (simpAdEx) model shown in Eq. 4 and 5 proposed Hertaeg and colleagues [44].

$$
\begin{gathered}
C \cdot \frac{\mathrm{d} V}{\mathrm{~d} t}=-g_{L} \cdot\left(V-E_{L}\right)+g_{L} \cdot \Delta_{T} \cdot e^{\left(\frac{V-V_{T}}{\Delta_{T}}\right)}+I-w=: w_{V}-w \\
\frac{\mathrm{d} w}{\mathrm{~d} t}=\left\{\begin{array}{cc}
0 & \text { for }\left|w-w_{V}\right|>\frac{\tau_{m}}{\tau_{w}} w_{V} \\
\Theta\left(V_{T}-V\right) \cdot\left[1-\frac{\tau_{m}}{\tau_{w}}\right] \frac{\mathrm{d} w_{V}}{\mathrm{~d} V} \frac{\mathrm{d} V}{\mathrm{~d} t} & \text { otherwise }
\end{array}\right. \\
\quad \text { if } V>V_{\text {up }} \text { then } V \rightarrow V_{r} \text { and } w \rightarrow w_{r}=w+b \\
\quad \text { if } w=\left(1+\frac{\tau_{m}}{\tau_{w}}\right) w_{V} \text { then } w \rightarrow\left(1-\frac{\tau_{m}}{\tau_{w}}\right) w_{V}
\end{gathered}
$$

1000 simpAdEx neurons were modelled with parameters randomly drawn from experimentally validated distributions and pairwise connection probabilities from literature [41]. Synaptic connectivities were simulated by conductance-based double exponential functions for AMPA-, GABA $\mathrm{A}^{-}$and NMDA-type synapses. Additionally, the synaptic models include short-term plasticity dynamics [59] with parameters drawn from separate normal distributions for short term facilitation, short term depression and a combined version of both $[35,84]$. A background current of $I_{\text {exc }}=250 \mathrm{pA}$ was applied 
to excitatory neurons and a current of $I_{\mathrm{inh}}=200 \mathrm{pA}$ to inhibitory neurons. For more details, see [41].

The model was supplemented with $\mathrm{GABA}_{\mathrm{B}}$-type synapses to simulate the slow IPSPs, which were found to be important within the state-space model [11]. The synaptic parameters for the conductance-based double exponential functions of GABA $_{B}$ were extracted from Golomb et al. [31] and presented in Table 5. The peak conductances of $\mathrm{GABA}_{\mathrm{A}}$ and $\mathrm{GABA}_{\mathrm{B}}$ were additionally reduced by multiplying with a factor of 0.3 to counteract the excess of inhibition within the network.

Table 5. Synaptic parameters of GABA . .

\begin{tabular}{lc}
\hline Synaptic Parameters & values \\
\hline$E_{\text {rev }}$ & $-90 \mathrm{mV}$ \\
$\tau_{\text {on }, \mathrm{GABA}_{B}}$ & $100 \mathrm{~ms}$ \\
$\tau_{\text {off }, \mathrm{GABA}}$ & $200 \mathrm{~ms}$ \\
$g_{\text {peak }, \mathrm{GABA} B}$ & $\frac{1}{5} \cdot g_{\text {peak }, \mathrm{GABA}_{A}}=0.2 \mathrm{nS}$
\end{tabular}

To test timing within the PFC model, the full network as shown in Fig. 1 within the gray box was stimulated in the beginning and in the end of time intervals with a step current of $I_{\mathrm{s}}=220 \mathrm{pA}$ for $10 \mathrm{~ms}$ to test various inter stimulus intervals $\overrightarrow{\Delta t}$. The model should ideally predict the presented inter stimulus interval. All simulations were performed with the NEST Simulator (version: 2.18.0 after extracting the parameters from MATLAB R2020a ([41]).

\section{Training the network}

After running the simulations, we extracted the states of all neurons $(N=1000)$ within a given time window $\Delta t_{w}$ following the readout mechanisms of a liquid state machine [48, 58] (cf. Fig. 1). Specifically, the states in the PFC network were calculated using a sum over spikes within a small time window during the second stimulation $\Delta t_{w}$ in which each spike is weighted using an exponential decay rate $\tau^{-1}$, see Eq. 6 .

$$
\mathrm{s}=\sum_{\substack{t_{i} \in \text { Spiketrain, } \\ \text { s.t. } t_{1}<t_{i}<t_{2}}} \exp \left(-\left(t_{2}-t_{i}\right) / \tau\right)
$$

To predict interval durations, a readout layer was added to the model with the number of output units $N_{r}$ matching the number of interval durations presented during training, cf. color-coded readout units in Fig. 1. Each output unit is then trained to be active for the respective time interval and to be inactive for all other time intervals. The readout weights were trained for all stimulus intervals and trials by maximizing the activity of the readout units for their corresponding stimulus intervals and minimizing for the remaining stimulus intervals.

Training of readout weights was realised by calculating the least squares to the expected targets, cf. Fig. 1. After training with 300 trials, the performance of time estimation was tested by presenting test intervals in $25 \mathrm{~ms}$ steps from $50 \mathrm{~ms}$ up to $750 \mathrm{~ms}$ and calculating the outputs of the previously trained readout weights. To predict the interval durations of the presented inter stimulus intervals, the negative output values were set to 0 , normalized by the sum of all readout neurons, multiplied by the respective training time intervals and summed across all readout units. The standard deviation of the estimated time was calculated over $N_{\text {test,trials }}=50$ test trials.

The hyperparameters that determine the states are the end point $t_{w_{2}}$, the length of the window $\Delta t_{w}$ of which spikes were extracted, and the exponential weight decay rate $\tau$. These parameters were selected using a grid search and optimizing for minimal root

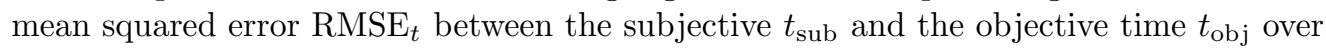


A

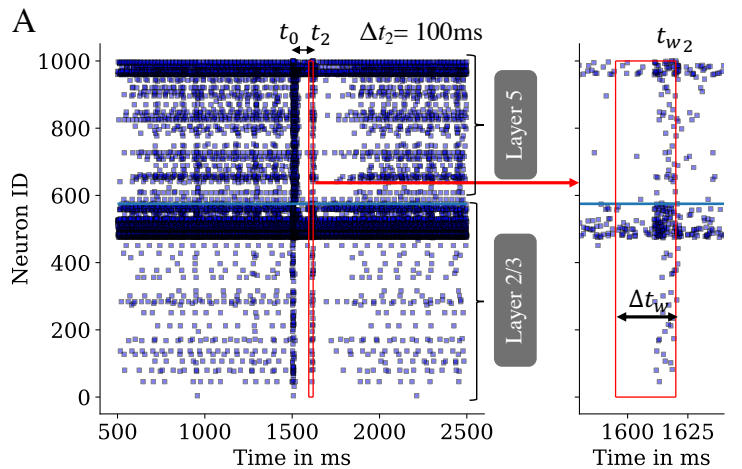

B

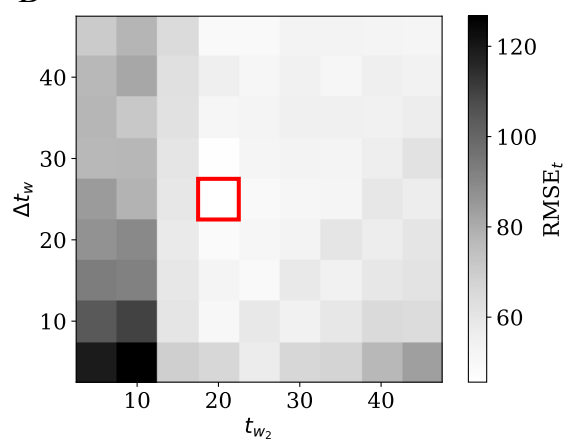

Fig 15. Extracting the states by defining a window. A. The rasterplot of all 1000 neurons (bottom: layer $2 / 3$ and top: layer 5) with stimulations at $t_{0}=1500 \mathrm{~ms}$ and $t_{2}=1610 \mathrm{~ms}$ for a ISI of $\Delta t_{2}=100 \mathrm{~ms}$ is shown here. The window to extract the states from the spiketrains is identified with the red box with an additional zoomed-in view. B. To find the optimal parameters for the window, a grid search was done with varying window size and end time. The best parameter set was selected by the minimum RMSE-value (red box).

a subset of training intervals $\left(N_{\text {train, trials }}=50\right)$ - see Fig. 15B. In a first coarse grid search for parameters $t_{w_{2}}, \Delta t_{w}$ and $\tau$, we found $\tau \rightarrow \infty$, effectively summing up the spikes within the window, to give lowest $\mathrm{RMSE}_{\mathrm{t}}$ for all settings of the other two parameters. Therefore, we fixed $\tau \rightarrow \infty$ and further optimized the two parameters $\Delta t_{w}$ and $t_{w_{2}}$ in a finer scale grid search depicted in Fig. 15B. The optimal parameter sets were selected by the minimum root mean squared error $\left\langle\mathrm{RMSE}_{t}\right\rangle_{\text {trials }}=45.6 \mathrm{~ms}$ with $\Delta t_{w}=25 \mathrm{~ms}, t_{w_{2}}=20 \mathrm{~ms}$ and $\tau \rightarrow \infty$ as indicated by the red box in Figs. 15A, B.

\section{Dopaminergic modulation}

The agonistic and antagonistic D2 receptor modulations were simulatad by modifiying various neuronal and synaptic properties. To alter the neuronal parameters, we extracted the changes from own unpublished data, in which the D2 receptor agonist quinpirole [22] was applied with a concentration of $10 \mu \mathrm{M}$. In contrast to that, the change of synaptic conductances was extracted from literature (for more details, see Table 6). Specifically, we interpreted the changes on neuronal and synaptic modulation under the application of dopamine as $100 \%$ agonistic modulation, cf. Table 6 , the properties without application of dopamine as $0 \%$ modulation and linearly interpolated between these two. Similarly, we extrapolated from $0 \%$ to $-100 \%$ to simulate the antagonistic modulation via dopamine. In order to simulate the acute change in neuronal activity induced by dopamine, we applied the modulation at test time without retraining the readout weights. To examine long-term plasticity-based adaptions induced by dopamine, we also retrained the readout weights in a second step for the case of $\pm 30 \%$ agonistic and antagonistic modulation.

\section{Noise}

To simulate variability across trials, an input layer of 10 Poisson neurons was added, such that each PFC neuron on average received input from one Poisson neuron with a firing rate of $1 \mathrm{~Hz}$. As a typical cortical neuron receives connections from approximately 1000-10 000 other neurons [6, 65], we examined the effect of higher input variability in our model by increasing the firing rates of our Poisson process to $1000 \mathrm{~Hz}$, thereby 
Table 6. Parameter alterations for D2 modulation.

\begin{tabular}{lccc}
\hline Parameters & & change & literature \\
\hline Neuronal parameters & & & \\
Reset potential & $\mathrm{V}_{\mathrm{r}}$ & $-5.2 \%$ & \\
Threshold & $\mathrm{V}_{\mathrm{th}}$ & $-3.7 \%$ & \\
Capacity & $\mathrm{C}_{m}$ & $+1.2 \%$ & \\
Leak conductance & $\mathrm{g}_{\mathrm{L}}$ & $-14.2 \%$ & \\
Leak reversial potenital & $\mathrm{E}_{\mathrm{L}}$ & $+6.6 \%$ & \\
Delta T & $\mathrm{sf}$ & $+27.8 \%$ & \\
Peak Potential & $\mathrm{V}_{\mathrm{peak}}$ & $-4.1 \%$ & \\
adaptation time constant & $\mathrm{t}_{\mathrm{cw}}$ & $-14.2 \%$ & \\
spike-triggeried adaptation & $\mathrm{b}$ & $+9.8 \%$ & \\
& & & \\
Synaptic parameters & & & \\
Peak conductance NMDA & $\mathrm{g}_{\mathrm{NMDA}}$ & $-20 \%$ & {$[54,74,90]$} \\
Peak conductance GABA & $\mathrm{g}_{\text {GABA }}$ & $-50 \%$ & {$[75]$}
\end{tabular}

emulating 1000 additional inputs. To test different noise levels, we increased the synaptic weights of the Poisson neurons, and in parallel reduced the background current to $(-7 \%,-21 \%,-36 \%,-50 \%)$ of the default to compensate over excitation. Specifically, for each synaptic weight factor, the background current was optimized using a grid search to minimize RMSE of averaged firing rates per neuron type and layer.

The time averaged sub-threshold membrane potential fluctuation, i.e. after removal of spikes, was shown to be at $4 \mathrm{mV}$ in experiments [17], which could already be reproduced within the PFC model [41]. For decreased background current and accordingly increased noise levels as described above, we find that sub-threshold membrane potential fluctuations are within the range of $2-4 \mathrm{mV}$.

\section{Description of analysis methods as needed}

\section{Rheobase}

The excitability of each PFC neuron was determined by the rheobase $I_{\text {rheo }}$ as proposed by Hertaeg and colleagues [44] as shown in Eq. 7.

$$
I_{\text {rheo }}=\left(a+g_{L}\right) \cdot\left[V_{T}-E_{L}-\Delta_{T}+\Delta_{T} \cdot \ln \left(1+\frac{a}{g_{L}}\right)\right]
$$

\section{Chaotic dynamics}

In order to determine whether our PFC model for time estimation is in the chaotic or non-chaotic regime, we slightly increased the stimulation current at interval onset and compared with the non-perturbed case. For this, we calculated the firing rate by convolving the spiketrains of each neuron with the causal kernel:

$$
w(\tau)=\left[\frac{\tau}{\sigma^{2}} \cdot \exp \left(-\frac{\tau}{\sigma}\right)\right]_{+},
$$

as described in [16], with $\sigma=50 \mathrm{~ms}$.

To determine the Lyapunov exponent, we then computed the euclidean distance between both per neuron firing rate trajectories as follows:

$$
d(t)=\sqrt{\sum_{i}\left(F_{i}(t)-F_{i, \text { pert }}(t)\right)^{2}},
$$


where $i$ denotes the neuron index. The Lyapunov exponent $\lambda$ is determined by the slope of the logarithmic distance shortly after the perturbation:

$$
\lambda=\frac{\log \frac{d\left(t_{2}\right)}{d\left(t_{1}\right)}}{t_{2}-t_{1}} .
$$

\section{Defining interval encoding pools}

In order to understand which intrinsic mechanisms were key features for the differentiation within training, we classified a neuron according to its highest readout weight contribution to its corresponding trained intervals as was already done in [11]. After classifying, the same neuron will not be considered for the remaining readout neurons. These neurons were sorted according to the corresponding interval encoding pool, see Fig. 6A. Only neurons with positive readout weights of above 0.1 are considered to examine the functional role of these neurons for training.

\section{Sequential activation of pools}

After defining the interval encoding pools, we examined whether there was a sequential activation of the pools from lower intervals to higher intervals. For this, we calculated the summed synaptic weights from the presynaptic lower intervals to a subsequent postsynaptic interval encoding neuron (cf. Fig 14A), normalized by the overall synaptic weight the neuron receives from all neurons and averaged over all postsynaptic neurons. The synaptic correlation activations between the pools were computed for all interval combinations and not only for the subsequent pools.

\section{References}

1. L. G. Allan. The perception of time. Perception \& psychophysics, 26(5):340-354 1979 .

2. L. A. Bizo, J. Y. Chu, F. Sanabria, and P. R. Killeen. The failure of weber's law in time perception and production. Behavioural processes, 71(2-3):201-210, 2006

3. D. A. Blankenship and N. H. Anderson. Subjective duration: A functional measurement analysis. Perception \& Psychophysics, 20(3):168-172, 1976.

4. D. J. Bobko, H. R. Schiffman, R. J. Castino, and W. Chiappetta. Contextual effects in duration experience. The American journal of psychology, pages $577-586,1977$.

5. M. Bortoletto, A. Cook, and R. Cunnington. Motor timing and the preparation for sequential actions. Brain and cognition, 75(2):196-204, 2011.

6. V. Braitenberg and A. Schüz. Anatomy of the cortex: statistics and geometry, volume 18. Springer Science \& Business Media, 2013.

7. C. D. Brody, A. Hernández, A. Zainos, and R. Romo. Timing and neural encoding of somatosensory parametric working memory in macaque prefrontal cortex. Cerebral cortex, 13(11):1196-1207, 2003.

8. A. Brown and S. Gershon. Dopamine and depression. Journal of Neural Transmission/General Section JNT, 91(2):75-109, 1993.

9. C. V. Buhusi and W. H. Meck. What makes us tick? functional and neural mechanisms of interval timing. Nature reviews neuroscience, 6(10):755-765, 2005 
10. C. V. Buhusi, M. B. Reyes, C.-A. Gathers, S. A. Oprisan, and M. Buhusi. Inactivation of the medial-prefrontal cortex impairs interval timing precision, but not timing accuracy or scalar timing in a peak-interval procedure in rats. Frontiers in integrative neuroscience, 12:20, 2018.

11. D. V. Buonomano. Decoding temporal information: a model based on short-term synaptic plasticity. Journal of Neuroscience, 20(3):1129-1141, 2000.

12. D. V. Buonomano. A learning rule for the emergence of stable dynamics and timing in recurrent networks. Journal of Neurophysiology, 94(4):2275-2283, 2005.

13. D. V. Buonomano. Neural dynamics based timing in the subsecond to seconds range. Neurobiology of interval timing, pages 101-117, 2014.

14. D. V. Buonomano and W. Maass. State-dependent computations: spatiotemporal processing in cortical networks. Nature Reviews Neuroscience, 10(2):113-125, 2009.

15. M. B. Cantor and J. F. Wilson. Temporal uncertainty as an associative metric: Operant simulations of pavlovian conditioning. Journal of Experimental Psychology: General, 110(2):232, 1981.

16. P. Dayan and L. F. Abbott. Theoretical neuroscience: computational and mathematical modeling of neural systems. Computational Neuroscience Series, 2001.

17. A. Destexhe, M. Rudolph, J.-M. Fellous, and T. J. Sejnowski. Fluctuating synaptic conductances recreate in vivo-like activity in neocortical neurons. Neuroscience, 107(1):13-24, 2001.

18. S. Droit-Volet. Time perception, emotions and mood disorders. Journal of Physiology-Paris, 107(4):255-264, 2013.

19. B. W. Dunlop and C. B. Nemeroff. The role of dopamine in the pathophysiology of depression. Archives of general psychiatry, 64(3):327-337, 2007.

20. D. Durstewitz. Self-organizing neural integrator predicts interval times through climbing activity. Journal of Neuroscience, 23(12):5342-5353, 2003.

21. D. M. Eagleman. Human time perception and its illusions. Current opinion in neurobiology, 18(2):131-136, 2008.

22. D. Eilam, I. Golani, and H. Szechtman. D2-agonist quinpirole induces perseveration of routes and hyperactivity but no perseveration of movements. Brain research, 490(2):255-267, 1989.

23. H. Eisler. Subjective duration and psychophysics. Psychological Review, 82(6): 429, 1975 .

24. H. Eisler. Experiments on subjective duration 1868-1975: A collection of power function exponents. Psychological Bulletin, 83(6):1154, 1976.

25. J. G. Fetterman and P. R. Killeen. Time discrimination in columba livia and homo sapiens.. Journal of Experimental Psychology: Animal Behavior Processes, 18(1):80, 1992 .

26. V. Franssen, A. Vandierendonck, and A. Van Hiel. Duration estimation and the phonological loop: Articulatory suppression and irrelevant sounds. Psychological research, 70(4):304-316, 2006. 
27. C. R. Gallistel and J. Gibbon. Time, rate, and conditioning. Psychological review, 774 $107(2): 289,2000$.

28. D. J. Getty. Discrimination of short temporal intervals: A comparison of two models. Perception \& psychophysics, 18(1):1-8, 1975.

29. J. Gibbon. Scalar expectancy theory and weber's law in animal timing. Psychological review, 84(3):279, 1977.

30. S. Glasauer and Z. Shi. The origin of vierordt's law: The experimental protocol matters. PsyCh Journal, 2021.

31. D. Golomb, X.-J. Wang, and J. Rinzel. Synchronization properties of spindle oscillations in a thalamic reticular nucleus model. Journal of neurophysiology, 72 (3):1109-1126, 1994.

32. S. Grondin. From physical time to the first and second moments of psychological time. Psychological bulletin, 127(1):22, 2001.

33. S. Grondin. Timing and time perception: a review of recent behavioral and neuroscience findings and theoretical directions. Attention, Perception, \& Psychophysics, 72(3):561-582, 2010.

34. S. Grondin. Unequal weber fractions for the categorization of brief temporal intervals. Attention, Perception, \& Psychophysics, 72(5):1422-1430, 2010.

35. A. Gupta, Y. Wang, and H. Markram. Organizing principles for a diversity of gabaergic interneurons and synapses in the neocortex. Science, 287(5451): 273-278, 2000 .

36. A. R. Halpern and C. J. Darwin. Duration discrimination in a series of rhythmic events. Perception \& Psychophysics, 31(1):86-89, 1982.

37. J. Hass and D. Durstewitz. Neurocomputational models of time perception. Neurobiology of interval timing, pages 49-71, 2014.

38. J. Hass and D. Durstewitz. Time at the center, or time at the side? assessing current models of time perception. Current Opinion in Behavioral Sciences, 8: $238-244,2016$.

39. J. Hass and J. M. Herrmann. The neural representation of time: An information-theoretic perspective. Neural Computation, 24(6):1519-1552, 2012.

40. J. Haß, S. Blaschke, T. Rammsayer, and J. M. Herrmann. A neurocomputational model for optimal temporal processing. Journal of computational neuroscience, 25(3):449-464, 2008.

41. J. Hass, L. Hertäg, and D. Durstewitz. A detailed data-driven network model of prefrontal cortex reproduces key features of in vivo activity. PLoS computational biology, 12(5):e1004930, 2016.

42. J. Hass, S. Ardid, J. Sherfey, and N. Kopell. Constraints on persistent activity in a biologically detailed network model of the prefrontal cortex with heterogeneities. bioRxiv, page 645663, 2019.

43. J. Henderson, T. A. Hurly, M. Bateson, and S. D. Healy. Timing in free-living rufous hummingbirds, selasphorus rufus. Current biology, 16(5):512-515, 2006. 
44. L. Hertäg, J. Hass, T. Golovko, and D. Durstewitz. An approximation to the adaptive exponential integrate-and-fire neuron model allows fast and predictive fitting to physiological data. Frontiers in computational neuroscience, 6:62, 2012.

. O. D. Howes, R. McCutcheon, M. J. Owen, and R. M. Murray. The role of genes, stress, and dopamine in the development of schizophrenia. Biological psychiatry, 81(1):9-20, 2017.

46. R. B. Ivry and J. E. Schlerf. Dedicated and intrinsic models of time perception. Trends in cognitive sciences, 12(7):273-280, 2008.

47. C. R. Jones, K. Rosenkranz, J. C. Rothwell, and M. Jahanshahi. The right dorsolateral prefrontal cortex is essential in time reproduction: an investigation with repetitive transcranial magnetic stimulation. Experimental Brain Research, 158(3):366-372, 2004.

48. J. Kaiser, R. Stal, A. Subramoney, A. Roennau, and R. Dillmann. Scaling up liquid state machines to predict over address events from dynamic vision sensors. Bioinspiration \& biomimetics, 12(5):055001, 2017.

49. R. Kanai, C. L. Paffen, H. Hogendoorn, and F. A. Verstraten. Time dilation in dynamic visual display. Journal of vision, 6(12):8-8, 2006.

50. S. Kaneko and I. Murakami. Perceived duration of visual motion increases with speed. Journal of Vision, 9(7):14-14, 2009.

51. K. Kang, M. Shelley, and H. Sompolinsky. Mexican hats and pinwheels in visual cortex. Proceedings of the National Academy of Sciences, 100(5):2848-2853, 2003.

52. J. Kim, A. H. Jung, J. Byun, S. Jo, and M. W. Jung. Inactivation of medial prefrontal cortex impairs time interval discrimination in rats. Frontiers in behavioral neuroscience, 3:38, 2009.

53. G. Koch, M. Oliveri, S. Torriero, and C. Caltagirone. Underestimation of time perception after repetitive transcranial magnetic stimulation. Neurology, 60(11): 1844-1846, 2003.

54. S. A. Kotecha, J. N. Oak, M. F. Jackson, Y. Perez, B. A. Orser, H. H. Van Tol, and J. F. MacDonald. A d2 class dopamine receptor transactivates a receptor tyrosine kinase to inhibit nmda receptor transmission. Neuron, 35(6):1111-1122, 2002.

55. H. Lejeune and J. H. Wearden. Vierordt's the experimental study of the time sense (1868) and its legacy. European Journal of Cognitive Psychology, 21(6): 941-960, 2009.

56. P. A. Lewis and R. C. Miall. Remembering the time: a continuous clock. Trends in cognitive sciences, 10(9):401-406, 2006.

57. P. A. Lewis and R. C. Miall. The precision of temporal judgement: milliseconds, many minutes, and beyond. Philosophical Transactions of the Royal Society B: Biological Sciences, 364(1525):1897-1905, 2009.

58. W. Maass, T. Natschläger, and H. Markram. Real-time computing without stable states: A new framework for neural computation based on perturbations. Neural computation, 14(11):2531-2560, 2002. 
59. H. Markram, Y. Wang, and M. Tsodyks. Differential signaling via the same axon of neocortical pyramidal neurons. Proceedings of the National Academy of Sciences, 95(9):5323-5328, 1998.

60. M. S. Matell and W. H. Meck. Cortico-striatal circuits and interval timing: coincidence detection of oscillatory processes. Cognitive brain research, 21(2): 139-170, 2004.

61. W. H. Meck. Affinity for the dopamine d2 receptor predicts neuroleptic potency in decreasing the speed of an internal clock. Pharmacology Biochemistry and Behavior, 25(6):1185-1189, 1986.

62. H. Y. Meltzer and S. M. Stahl. The dopamine hypothesis of schizophrenia: a review. Schizophrenia bulletin, 2(1):19, 1976.

63. H. Merchant and V. De Lafuente. Introduction to the neurobiology of interval timing. Neurobiology of interval timing, pages 1-13, 2014.

64. C. Miall. The storage of time intervals using oscillating neurons. Neural Computation, 1(3):359-371, 1989.

65. J. M. Murre and D. P. Sturdy. The connectivity of the brain: multi-level quantitative analysis. Biological cybernetics, 73(6):529-545, 1995.

66. H. Okamoto and T. Fukai. Neural mechanism for a cognitive timer. Physical Review Letters, 86(17):3919, 2001.

67. H. Onoe, M. Komori, K. Onoe, H. Takechi, H. Tsukada, and Y. Watanabe. Cortical networks recruited for time perception: a monkey positron emission tomography (pet) study. Neuroimage, 13(1):37-45, 2001.

68. J. J. Paton and D. V. Buonomano. The neural basis of timing: distributed mechanisms for diverse functions. Neuron, 98(4):687-705, 2018.

69. I. Polti, B. Martin, and V. van Wassenhove. The effect of attention and working memory on the estimation of elapsed time. Scientific reports, 8(1):1-11, 2018.

70. S. Rahman, J. Zhang, E. Engleman, and W. Corrigall. Neuroadaptive changes in the mesoaccumbens dopamine system after chronic nicotine self-administration: a microdialysis study. Neuroscience, 129(2):415-424, 2004.

71. T. Rammsayer. Temporal discrimination in schizophrenic and affective disorders: evidence for a dopamine-dependent internal clock. International Journal of Neuroscience, 53(2-4):111-120, 1990.

72. T. Rammsayer, P. Netter, and W. H. Vogel. A neurochemical model underlying differences in reaction times between introverts and extraverts. Personality and Individual Differences, 14(5):701-712, 1993.

73. J. Reutimann, V. Yakovlev, S. Fusi, and W. Senn. Climbing neuronal activity as an event-based cortical representation of time. Journal of Neuroscience, 24(13): 3295-3303, 2004.

74. J. K. Seamans and C. R. Yang. The principal features and mechanisms of dopamine modulation in the prefrontal cortex. Progress in neurobiology, 74(1): $1-58,2004$. 
75. J. K. Seamans, N. Gorelova, D. Durstewitz, and C. R. Yang. Bidirectional dopamine modulation of gabaergic inhibition in prefrontal cortical pyramidal neurons. Journal of Neuroscience, 21(10):3628-3638, 2001.

76. G. B. Smith, B. Hein, D. E. Whitney, D. Fitzpatrick, and M. Kaschube. Distributed network interactions and their emergence in developing neocortex. Nature neuroscience, 21(11):1600-1608, 2018.

77. R. Spanagel and F. Weiss. The dopamine hypothesis of reward: past and current status. Trends in neurosciences, 22(11):521-527, 1999.

78. S. Stevens. Psychophysics: Introduction to its perceptual, neural, and social prospects. 2000 ed. New York: John Willey and Sons, 1975.

79. S. Thönes and D. Oberfeld. Time perception in depression: A meta-analysis. Journal of Affective Disorders, 175:359-372, 2015.

80. N. Ueda, K. Maruo, and T. Sumiyoshi. Positive symptoms and time perception in schizophrenia: A meta-analysis. Schizophrenia Research: Cognition, 13:3-6, 2018.

81. G. E. Uhlenbeck and L. S. Ornstein. On the theory of the brownian motion. Physical review, 36(5):823, 1930.

82. V. Van Wassenhove, D. V. Buonomano, S. Shimojo, and L. Shams. Distortions of subjective time perception within and across senses. PloS one, 3(1):e1437, 2008.

83. K. Vierordt. Der zeitsinn nach versuchen. H. Laupp, 1868.

84. Y. Wang, H. Markram, P. H. Goodman, T. K. Berger, J. Ma, and P. S. Goldman-Rakic. Heterogeneity in the pyramidal network of the medial prefrontal cortex. Nature neuroscience, 9(4):534-542, 2006.

85. J. H. Wearden, H. Edwards, M. Fakhri, and A. Percival. Why" sounds are judged longer than lights": Application of a model of the internal clock in humans. The Quarterly Journal of Experimental Psychology: Section B, 51(2):97-120, 1998.

86. H. Woodrow. The reproduction of temporal intervals. Journal of Experimental Psychology, 13(6):473, 1930.

87. H. Woodrow. The temporal indifference interval determined by the method of mean error. Journal of Experimental Psychology, 17(2):167, 1934.

88. M. Xu, S.-y. Zhang, Y. Dan, and M.-m. Poo. Representation of interval timing by temporally scalable firing patterns in rat prefrontal cortex. Proceedings of the National Academy of Sciences, 111(1):480-485, 2014.

89. A. D. Yarmey. Retrospective duration estimations for variant and invariant events in field situations. Applied Cognitive Psychology: The Official Journal of the Society for Applied Research in Memory and Cognition, 14(1):45-57, 2000.

90. P. Zheng, X.-X. Zhang, B. Bunney, and W.-X. Shi. Opposite modulation of cortical n-methyl-d-aspartate receptor-mediated responses by low and high concentrations of dopamine. Neuroscience, 91(2):527-535, 1999. 